\section{COMMISSION EXTRAPARLEMENTAIRE DES FORCES HYDRAULIQUES}

\section{Textes des Procès-Verbaux des Séances}

\author{
SÉANCE DU 31 MAI 1917 (4 Séance)
}

Présents : MM. Klotz, Président ; Develle, Monestier, Bérard, Lovrties, Léon Perhier, Rousseau, du Longbors, Mahieu, Salues, Dabat, Troté, Blazeix, Chammem, Gamllard, Conte, Monsarrat (remplaçanl M. Dupoñteit) Camen, Berthelemy, Hrtier, Lavaud, Bovcinyen, Conden, Tissenand, Pinot, Bougault, Petit, Lontie. Excusés : MM. Bedouge, Jusy.

La séance est ouverte à Io h. $1 / 4$.

M. re Présinent. - La parole est à M. Margaine.

M. Margarne. - Messieurs, l'exposé que j'ai à faire sera un peu différent de ceux qui ont été faits jusqu'ici. J'ai à vous expliquer les idées qui ont conduit quelques membres de la Commission des Travaux Publics de la Chambre à rédiger le projet tel qu'il a été déposé.

La Cómmission des 'Iravaux Publics s'est trouvée on face du projel revena du Sénat dans la législature précédente: Ce projel a été mis en discussion à la Commission et un certain nombrc de membres ont pensé que les principes sur lesquels le projet ćtait bașé n'avaient pas suffisamment de solidité sociale, quil ćtait nécessaire de leur en domner. Et; à partir dü moment où jls sont entrés dans celte voie, ils ont été conduits, de proche cn proche, à introduirc les principes qui sont expliqués dans l'exposé des motifs du projet en question.

Mon collègrue Bedouce n'a pas pu venir aujourd'hui, mais il m'a chargé de vous dire qu'il adhère entièrement, ainsi que M. Mistral, aux idées que je vais essayer de vous exposer dans lous leurs détails.

Dans le projet qui lui avait été soumis, la Commission des Travaux Publirs voulut revendiquer le droit de concession, majs il ne lui en était donné aucune justificationn. Il y était dit qu'on ne poivait construire de chutes qu'ávec la permission de l'Etat. Jusqu'ici, Messieurs, l'Etat r'a jamais usé que de droits de police et il nous semble que dés droits de police ne sont pas suffisants pour édicter l'obligation de la concession.

Il est évident 'que, lorsqu'un ritoyen s'installe, il se trouve en présence des différents droits des voisins : l'Etat doit intervenir pour que ces droits soient respectés et que ce citoyen se fasse sa place conformément nux lois. Mais, à partir du moment où la place esi faite, l'Elat a épuisé ses droits de police. I] n'y a aucune raison pour qu'il dise à l'individu qui s'est. installé qu'il ne restera la quc pendant un certain lernps et il y a encore moins de raisons pour qu'il lui dise : " au hout de ce temps, quand vous partirez, vous me laisserez gratuitement ce que vous avez inslallé ". Si l'on admettait que la concession se fasse simplement par la volonté du Prince, Messieurs, ce ne seraient plus des prinicipes républicains. mais des principes de tyrannie: On pourrait aussi an dire que l'industrie des métaux, la culture de la betterave, Ii. production du sucre, ne se feronl que par concession.

Il fant au droit de concession une has sociale. Nous lavons trouvée dans le principe de la domanialité publique et nous sommes allés rechercher la base de re principe dans les idées qui onl été émises an moment de la Rérolution de $8 \mathrm{n}$ et qui ont ćté crimplètement oubliées par l'Fmpire. Nous avons rappelé qué, ail moment de l'Assemblée nationale, on avait ronsidéré le domaine public comme étant, en laif. la richesse nationale. On ne consiclérail fins que lc domaine public fut une chose matérielle, rinsi quie J'idée s'en est laite peu à peu : vous n'avez qu’à relire le décret relntif ain domaines nalionaux ( $2 \mathrm{r}-22$ novembre 1700$)$ nour voir que celle question de matérialité n'était pas du tout envișagée :

Voilà l'article $\mathrm{I}^{\text {ar }}$ : "Le domaine national proprement dit " s'entend" de loutes les propriétés foncières et de tous les droits " réels ou mixtes qui appartiennent à la nation, soit qu'elle en
" ait la possession et la jouissance actuelles, soit qu'elle ait seu" lement le droit d'y entrer par voie de rachat, droit de réversion " ou autrement ".

G'est done, en quelque sorte, le fonds commun de richesse publique qui constitue le domaine public de sorte que, lorsque vous diles que l'Etat a le droil de concéder sur les rivières navigables parce qu'il est propriétaire, nous disons que l'Etat a le droit de concéder la force hydraulique contenue dans ses rivières parce que cette force in partie du domaine public. Il n'y a pas de contestation en raison du caractère de navigabilité des rivièreś navigables, mais il nien est cependant pas moins nécessaire de reconnaître l'assise du droil. C'esl l'usage du domaine public que l'Etat autorise par voie de concession.

On nous oppose la question de la propriété. On dit : “ Vous touchez ì la propriélé ; ces moulins, ces usines; au moment de la Pévolution de 8 , ont été considérés conme des propriétés particulières, par conséquent ils restent pröpriétés particulières i). lo ne suis pas très sûr que la base soit absolue. Je ne cóntesto pas qu'on les ait considérés à ce momént comme des propriétés particulières ; on n'avait aucune raison, à celte époque-là, dé considé:er les moulins comme rentrant dans de domaine public. Ei, pourtant. je suis bien sûr gite'si la Révolution avait pu ce qui ćail impossihle - prévoir le ròle ultériem de la houille lanche, elle n'aurait pas manqué d'incorporer les moulins daus le domaine public. Il y a eu un moment où le législateur a eu un momenl d'hésilation, cependant. Ces moulins avaient alor un rôle commun. c'élaient des " banalités », et les " banalités ), toul au début de la Révolution, avaient été réservées. On se demandait s'il n'y avait pas là quelque chose sur quoi il fallait conserver l'autorité ; loreque vous prenez la loi du is mars $179^{\circ}$ sur la destruction dix régime féodal, vous trouvez deux articles que je vous demande la permission de lire :

AnT. 23. - Tous les droits de banalités, de, fours, moulins, pressoirs, boücheries, taureaux, verrats, forges et autres, ensemble les sujétions qui "y sont accessoires.... sont abolis et supprimés sans indemnité, sous les séules exceptions ci-après :

Art. 24. - "Sont exceptés de la suppression ci-dessus et seront rachetables : $\mathrm{r}^{\circ}$ les banalités qui seront prouvées avoir été établies par une convention souscrite entre une communauté d'habitants et un particulier non seigneur ; $2^{\circ}$ les banalités quil sont prouvées avoir été établies par une convention souscrite par une communauté d'habitants et son seigneur, et par laquelle le seigneur aura fait à la communauté quelque avantage en plus que de s'obliger à tenir perpétuellement en état les montures, fours et autres objets banaux $; 3^{0}$ celles qui seront prouvées avoir eu pour cause une concession faite par le'seigneur à la communauté dès habitants, dës droits d'usage dans les bois, des prés, ou de communs en propriété.

Art. 26. - « Il est fait défense aux ci-devant barriers d'attenter à la propriété des moulins, pressoirs, foứs et autrés 'objets de lá banalité, desquels ils sont affranchis par l'art. 25 ; ladite propriété est mise sous la sauvegande de la loi et il est enjoint aux municipalités de tenir.la main à ce qu'elles soilent respectées jo

La Révolution avait envisagé que l'usage commun avait besoin de la protection de la Ioi et, si cette idée a disparu, si on a laissé les usagers revendiquer la pleine propriété de ces petits moulins, c'est que les principes de liberté auxquels on était attaché ont eu pour conséquence l'apparition d'autres moulins, de sorte que le rôle collectif des moulins banaux s'est complètement évanoui Les moulins sont redevenus propriétés privées : la domanialité n'avait plus aucune espèce de raison d'être en ce qui les concerne.

Les principes de domanialité n'ont pas disparu complètement pour cela. La Révolution n'a continué à légiférer, en matière générale, que sur ce qui lui est apparu comme richesse nationale. Avant elle, des tentatives avaient été faites dans cette voie je ne vous ferai pas perdre votre temps à vous rappeler le texte qui, sous Colbert, a rapproché la production des forêts de la richesse nationale. Le fameux édit de Colbert a été un des attentats à la propriété privée, les plus forts qu'eût commis la royauté contre la liberté individuelle du propriétaire par lobligation de 
suivre certaines méthodes dexploitation des bois privés, cela en verlu de lá simple raison que te pols laisail la lortune du rays.

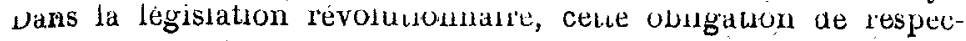
ter une propriété générale comme les forêts ne put s'étendre en raison du guand service de celle epoque et lanre unsparaitre loute illeinte a la hverte inuvrauene. wile ne devalt réapparaitre, tout al noms en lentaluve, dans le dornaine public, qu'un siècle après lil grande hevolulıon, à l'époque acıuelle, je ne dis pas, Messleurs, à la velde de la luture Révolution.

Pous nous, tressieurs, le domaine public est constitué par les sources de la richesse nationale; cest le fond commun de rehesses quaucun choyen 11 a ciece el qui appartient a tous, non pas indivauellenent, et lous peuvent en lare usage, comme un

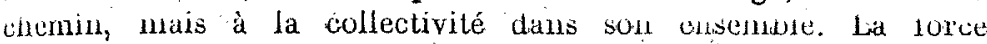
hyraulique a ce caractère parce qu'ale nest là création d'aucun tiluyen : elle est engendrée par la pesanteur. Llie lail partie du tomaine public el nous ne pouvons autolser un citoyed à lexploiter que par rone de concession, parce que c'est la nation, c'est la collectivite qui en a la garde. roila uonc te principe de la concession établi.

Partant de ce principe, de la concession, nous arrivons aux conditions de la concession.

Ces conditions de la concession sont, naturellement, que le amaine public ne doil ètre exploité que dans lintérêt dé tous.

aut que ce soit l'intérèt commun qui bénéficie de l'exploitalion du domaine public : nous arrivóns au principe des rederances. Il laul que lébénélice tiré du dómaine public aille en partie, lùt-ce en faible partie, à l'Etat, non pas comme impôt, non pas comme contribution aux depenses publiques, mais comme part de l'exploitation du domaine public.

Mais, il y a plus. Il y a plus que la simple redevance. Il y a uncore le bénétice commun collectif au point de vue du développement total de la nation et de l'évolution générale de l'indusirle. Je vous demande la permission de vous rappeder ce qui se passe dans d'autres pays.

11 n'est pas un seul de vous qui n'ait entendu parler des grands "Lusts " d'Aménque, la "Standard Oil ", la "Steel cooperative ", l' "Amalgamated ", la " General blectric », etc... Cie qu'on sail moins, cest que ces grands trusts contre lesquels on tenta en vain de lutter aux Etats-Unis, non seulement forment des puissances isolées, mais se sont agrégés entre eux. Ces agregations nous sonl comnues par les attaques d'une certainc presse américaine et des enquêtes qui en furent la conséquence. If y a peu de temps, au cours de la fameuse enquête du " Money lrust ", on a mis à jour que les financiers d'Ámérique avaient. constilue un groupe bancaire comprenant $70 \%$ de la puissance bancaire totale ef qu'autour de ce groupe sont venus se réuntr les grands trusts dont j'ai parlé: On trouve en Amérique, formant te centre de celle agregation, seulement six banques à la tête desquelles se-trouve un homme que tout le monde connait, c'est Horgan; le chet de la banque Morgan, de New-York, de la banque Morgan, de Londres, de la banque Drenelle, de Philadelphie of la biluque Morsan; Harjes, de Paris. Ces six banques ont échangé des administrateuris avec des banques intemationales, avec les grands irusis que jai cités, de sorte que les chefs de ces. diverses corporations ont fini par occuper :

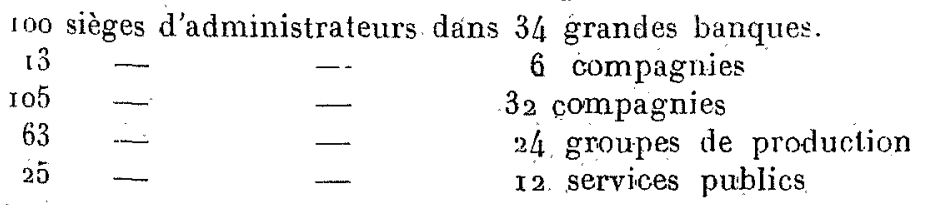

soit $34 \mathrm{x}$ sièges dans rr 2 sociétés. Si je. prends seulement les forces hydrauliques, celles qui nous intéressent plus particulièrement, je vois qu'elles se répartissent en sept groupes dont la "General Electric Company " produit à elle seule autant que les six autres réunis.

Yoilà une concentration considérable qui se fait en Amérique, qui est tellement considérable que la population après s'en être tout d'abord émue au point do vue intérieur, a fini par y reconnâitre une arme utile, en sorte qu'il s'est formé un mouvement d'opposition contre ceux qui lont attaquée el que l'on arrive maintenant à considerer que, lom de sopposer a cette sorte de puissance qui s'élève, on doit, au contraire, la domestiquer el sen servir pour la puissance ue 1 Amerique. A theure actuelle, se discute au Congrés un anendement qui consiste à soustraire

í loi contre les trusts (Sherman Ach tout ce qui est commerce à 1 erianger ou comnerce a exporlanon. 1 ar ailleurs, la cour supreme qui, $11 \mathrm{y}$ a quinze jours à peine a decidé d'ajourner loutes les poursuiles intentées contre les divers trusts. Cela est le résultat aun mouvenent dopinion qui se produit en ce moment: et qui pense qu'il convient de réglementer ces trusls pour sé plus grand bien, la plus grande lorce du pays, el non pas de les délruire.

Vous avez constali, en Allemagne, quelque chose de semblable, $\therefore$ organisations comme la "Metallgesellschaft " ou lat "Badis.a Anilin " qui cherchent ù étendre la puissance allemande an dehors el à s'assurer le monopole des marchés commerciaux. Bien loin de les combattre, 1 Ltat allemand les soutienl, mais le règlement a fail le syndical forcé de la production de la potasse pour obliger les Américains à cesser leur concurrence el a dissous par là mème le syndical de libre produclion qui s'élait formé. récemment, il a juslıtué le Syndicat obhgatoire des llines qu survivra da la guerre. Voila, hessieurs, des organisations cons1tćrables : 11 laut s'en préoccuper el tàcher d'en créer dans notre pays pour pouvvir luller à armes egales avec létranger.

Kemarquez, Messieurs, que l'énergie liydraulique vise à la proúction de matières qui ne peuvent pas ćchapper à la loi de concentration donl $j$ 'ai parlé toul à l'heure. Lin ce qui concerne les produits de l'électro-métallurgie, il est impossible que vous en puissiez laisser le marché entièrement libre, il est trop restreint ; on est obligé d'arriver à laire des syndicals internationaux el il est impossijle que ces syndicats se constituent si l'Elat n'y entre pas, el il le tera, non seulement de par' ses droits de police, mais de par le lait que c'est lui qui concède les usines. il doit, non seulement délendre ses organisations, mais encore les aider à régler la consommation el le marché. Voilà le point de départ des conditions de la concession.

Nous avons distingué deux séries d'usines : les usines les plus puissantes, celles qui seronl les maîtresses du marché. Pour celles-là, nous demandons aux industriels d'admettrc des reprósentants de l'Etat à côté d'eux, dans leurs conseils. Né croyéz pas que nous ayons l'intention de leur envoyer des ingonicur's pour les aider à diriger l'usine elle-même : elle ne sera jamais inicux dirigée que par les industriels. Ce que nous demandons. c'est qu'à partir du moment où une usine commence à régles. le marché, l'Etat ait des représentants à côlé des industriels pour s'associer à ce qu'ils font, pour leur indiquer la voie la meilleure dans l'intérêt public. Que les industriels qui résistent à ses idées mé permettent de leur dire que si cette organisation avait existé, laffaire des carbures ne se serail pas produite parce que les rprésentants de l'Etat auraient vu cé qui se passáil, ils auraient pu dire à certains industriels : "Vous allez trop loin ", et lorsque to pouvoir judiciaire, aveuglć par certaines circonstances, est allé un peu loin dans la voie de la répression, les représentants de l'Etat auraient pu dire « prenez garde, c'est vous, maintenant, qui allez trop loin ". De tels représentanis de l'blat re peivent ọtre que des techniciens; il est nécessaire que le ruprésentant de l'Etat puisse comprendre les intérèts des industriels comme l'inlórêt de la collectivité. Nous aurons donc des usines dans lesquelles il y aura un représentant de ]'Etat ; nous n'en demandons pas dans toutes. Il y aura un nombre considérable de concessions. i) accorder : nous ne pouvons pas avoir un représentant dans. chaque usine. Nous distinguons donc deux catégories :

r. Usines très puissantes, de plus de ro.00o kilowatts où nous serons représentés :

$2^{\circ}$ Usines de moins de Io.00o kilowatts pour lesquelles nous nous contentons de prévoir une redevance a titre de part d'exploitation du domaine public.

A ces usines plus faibles, nous ne pourons pas donner tout ce qui a été réclamé, notamment, nous né pouvons pas leur donner le pouvoir d'expropriation. Nous cherchons a leur donner le 
maxımum de ce qu'il faul pour qu'elles puissent s'installer saus perdre lour caractère dintérèt privé. Hlles auront les droils que leur confèrent aujourd hui les lois, avec ceci en plus que les donmages que leur. lonctionmement pourrait causer ne pourra pas donner le droil de les laire cesser, mais seulement celuı d ètre .alemnisé.

Mus navons pas proposó de leur domer le droil dexpropria...nn. pour celte raison quil y aura beaucoup de ces indusiries yai ont un caractere tellement privé que les particuliers ne corniprendraient pas quon puisse leur octroyer le droil d'expropriacion. Supposons que, dans une petite commune, sétablisse un labricant de chapeaux de f'eutre ou de bometerte : on the comprendrait véribablement pas que ce labricaul abtienne te 11:uil d'expropriation pour extreer son industrie.

Au contrairc, aux usines de plus de ro.00o kilowatts, usines tres importantes, nous domnons toutes les laveurs que les lois prévoichl pour rompre les ubstacles à un facile élablissement. blles muront, non seulement le droit dexpropriation, mais tous les droils qui sont ochoyés aux concessionnaires de travaux pu-

$\therefore$. Les ouvrages sonil des ouvrages de grande voirie : elles ont les serviludes dé passage. Ce sont des droils qui peuvent paraître exorbilants ; je me permettrai de rappeler que, dans lihstoire, on a dù quelquefois pour favoriser cerlaintes industries, leur donner des lroils qui paraissaient exagérés. Rappelerai-je les privileges donnes ì l'industric de la verreric sous l'ancien régine : A ehrique époque correspondent des layeurs spéciales el, pour "rivelopper les usines particulières anxquelles nous nous intéres. sons, nous lon donnons les faveurs dont j'ai parlé:

En re qui concelme le représentant de l'Etat, si hous avous prévu des ingénienirs des travaux publics, ce nest pas que nons preferions tel on tel ministore. Ce que nous désirons, e'est un technicien fui ait denx qualites : dabord l'esprit de tradilion, puis, superposé à cel espril de tradition, un esprit d'évolution, parce fute le premier sans lo second n'est autre chose que la routine.

Dirons-nous que les ingénieurs de l'Etat possedent complètement les qualilés dont je viens de parler fe the hàte de répondre : non. Il s'en faut de beaucoup qu'ils possèdent ces doux qualités, mais nous avons pensé que célait conx qui potirraient les acquérir le plus facilement.

Lorsque la Convention a établi les ingénieurs dEtal, il fant voir comment elle a procédé. thlle a vouhu que ces ingénients I'liat eussent, au début, une hante culture scientifique. Pensezvous, Messicurs, que cétail dans le bit davoir des savants : l.irl de la construction ne comportait pas alors des diflicultés scientifiques tellement considérables, au contraire. La Convention avait soulement pensé que cette haute culture scientifique était susceptible de développer chez eux l'esprit d'évolution el de progrès jour la recherche conslante des procódés les moilleurs. Quan a l'esprit de tradition, il s'est créé du fail que ces ingénicurs formaient $11 \mathrm{n}$ corps ; pent-ìtre l'espril de progrès a-t-il un peu disparu chez ces ingénieurs, c'est parce qu'ils ont fini par so confiner dans des simples services d'entretien. Si on. avait eu la sagesse de domner ces services d'entrelien à des fonctionnaire: liuts prur cela, yui aient un bagage scientifique moins considé rable pent-elre, mars d'une instruction pratique plus grande, les ingénieurs y auraient gagné et répondraient aux qualités dont j’ai parlé plus haut.

En tous cas, ce sont cux qui auront le moins à faire pour les acquérir, et c'est pour cola que nous avons prévu dans notre projet de loi l'emploi des ingénicurs de l'Etal - non pas forcément ceux des Travanx Publics - comme devant venir à côté des industriels pour chercher à développer, à conduire l'industrie hydraulique prise en général, et non pas chaque usiné prisé en particulier: Voila pour les usines futures.

Il nous reste à parler des usines actuelles.

Celles-la, Messieurs, ont acquis un certain droit de propriété que nous ne pouvons pas leur contester. Nous disons qu'elles sont installées sur le domaine public, mais, au moment de leur b́ablissement, personne ne le leur a dit. Elles ont cru qu'elles Giaient chez elles et il faut les considérer, par conséquent, comme ¿tant en possession d'une véritable propriété. Que faisons-nous - ous tenons à respecter les prancipes - Lout socralistes que soient cerlains d'entre nous - nous les rachetons en disanl : "voiro concession élait de $7^{5}$ ans. rat conséquent, nous vous prenons rotre proprété, non pas aujourd hui, mais dans 75 ans. Nous Illons donc vous verser aujourd hui le capilal qui est necessarc pour constituér, en $7^{j}$ ans, la valcur de vole usine. vous 1 placerez, comme vous voudrez, el, dans $7^{\hat{j}}$ ans, hous hous trou verons avoir racheté voute usine, elle sera à nous ". De cette sorte que, dans $7^{5}$ ans, celle usine se trouvera dans les mèmes cuditions que celles qui arrivent in lin de cessation et te regane nera unitorme pour toutes les usines de france.

Insisterai-je, nessieurs, sur dautres dispositions is Je ne crois pas utile d'entror uans le delall. Il $\mathrm{y}$ a seulement un point sur lequel j'attire volre attention.

Un a beaucioup parlé de la redevance locale. Nous n'en avirs . is prévu; el. cest volontairement.

Nous pensons qu on il a pas ä rélablir, contre ces industries qui naissent, la dime. Autuefois, il fallail payer la dime pour s installer chez le seigneur. Nous estimons que ces usages ont nsparu, qu'il ny a pas de raison pour laire payer par bes usines une dime aux localités. Au contraire, elles constitueront une nouvelle richesse pour les pays où elles sinstalleront. Pourquoi payeraient-elles une dime qui augmenterait leur prix do revient ? Pourquoi domer gratuitement une partie de la lore produite. En retour de quoi:

Lulin, nous avolis parlé d une "Commission " el non pas d'un "Office " parce que nuus restons convaincus que les organisations de direction adminstrative n'ont de valeur que si elles sont dirigées par des individualités. Il l'aul que la direction genérale appartientie ì un seul homme, non pas à un onsemble hommes : les responsabilités s'y dissoudraient. Il faut qu'il y: ait un bon Directeur, qui ait à côlé de lui un Conseil dont 11 soit tenu de prendre l'avis, mais qui agisse dans la mesure quil voudra, et qui soit responsable.

Pour éviter aussi les dilficultés dont on s'est plaint en ce qui concerne les délais' de procédure, nous avons fixé des délais au bout desquels, si l'Administration na pas répondu, le demandeur' pourra se considérer comme étant en possession d'un titre aná logue au dernier accordé pour une industrie semblable. L'Administration pourra, si elle le veut, prévenir qu'un délai plus long: lui sera nécessaire, mais elle devra dire loquel, el, lorsqu'il serił expiré, les lormalités devnont ètre terminées, ou elle serà forcluse.

Voilà, Messieurs, les principes dont nous nous sommes inspirés. J’ai essayé de rous les exposer avec le plus de clarté poṣshle. Vous m'excuserez si je n'y suis pas parvenu.

M. Le Pkésident. - Nous vous remercions. La parole est a M. Petit.

M. Petrt. - Messieurs, des auditions auxquelles il a été procédé, des explications qui ont été données, il résulte que tonl lẹ monde est d'accord sur la volonté d'aboutir ainsi que sur les buls is atteindre. Je pense qu'il sera relativement facile d'arriver à the entente complète el rapide sur" les moyns à adopter pour. réussir.

Il est cependant un point sur lequel l'accord ne s'est pas fail, c'est la question du rattachement à un ministère déterminé. Je voudrais écarter tout de suite cette question dú débat. Car d'une part, les principes à discuter sur les questions juridiqués sont beaucoup plus importants que cette question du rattachement à un ministère. D'autre part, il paraît logique de s'entendre sur la création de I'organe à instituer avant de voir à qui il sera attri: bué. C'est pourquoi je vous proposerai, après avoir affirmé le principe de l'unité de direction, de réserver la question de lattribution à un ministère déterminé et, par cela mème; de réserver la question connexe de savoir si cette attribution devra être défnitive ou s'il $y^{\prime}$ aura lieu de prévoir d'abond une attribution temporaire pour le temps de guerre, puis une attribulion définttive pour le temps de paix.

Je passe à l'examen des projets.

Cies projets peuvent êtrè divisés en deux groupes partant de conceptions différentes : 
$I^{\circ}$ Projel de l'armement ; $2^{\circ}$ Tous les autres projets.

Le projel de l'armement vise exclusivement de temps de guere of exclusivement les cours d'eau non donianiaux.

les autres projels visent à létablissement d'un régime delinilif et statuent pour tous les cours d'eau, domaniaux et non domaniaux.

Le projel de l'Armement a un avantage : sa simplicité. Il lient ch quatre articles donl le vote devrait permettre dutiliser les chules d'eau pour nos productions de guerre. A mon avis, oil peul y faire cetle sérieuse objection qu'il ne donne qu'une solution partielle el temporaire, qu'il ne statue que pour le lemps de guerre. Or, M. le Président du Conseil est venu nous dire avec une grande force que la question est en suspens depuis 23 ans et qu'il faut aboutir. Serait-il conforme au vou qu'il a exprimé, de ne statuer que pour le temps de guerre ?' Peut-on atmethe que dans quelques mois, au lendemain de la paix, on retombe dans le néant ; peut-on admettre qu'après quelques mois d'un régime temporaire on vienne dire au pays : rien n'est fait ; nous commençons la $24^{\circ}$ année d'études el de projets ? Pour moi, jo ne le crois pas. Quoi qu'il en soil, ce sera une des premieres questions que la Commission aura à trancher.

Les autres projets sont très voisins les uns des autres. Dans chacun d'eux, on retrouve les mêmes' directives, et cela se comprend puisqu'ils sont la conséquence, l'aboutissement logique de tous les projets qui ont été élaborés successivement pendanl 23 alls. On a profité de l'expérience acquise et on est arrivé, dans les différentes administrations, à des projels fort peu différents. Los principes sonl les mèmes; il n'y a guère de divergences que sur des questions d'ordre secondaire. Il semble donc qu'on arrivera rapidement à se mettre d'accord sur les principes, et sur un texte.

If y a cependant un point sur lequel j'attire l'attention de la Conmission parce que je le considère comme important. La plupart des projets qui nous sont soumis ont un caractère commun qui me parail, à l'heure acluelle, n'être pas sans inconvénients sérieux : ils sont généralement fort longs. L'un a 74 articles, l'auties ont moins d'articles mais plus d'alinéas, ils comportent de 60 à 80 dispositions différentes; je ne crois pas bon d'obliger ainsi le législateur à entrer dans lès détails d'application ; le législateur a pour mission de poser des principes ; les détails d'application sont du domaine des règlements d'administration publique.

Je voudrais, si la Commission me le permet, lui lire quelques lignes du cours de M. Ducrocq, un de nos maîtres en droil public :

"Le pouvoir légíslatif se contente de poser des principes el laisse au pouvoir exécutif́ le soin de statuer sur la misc en couvre. Portalis, dans son discours sur le titre préliminaire du Code civil a dit : "L'office de la loi est de fixer par de grandes vues les maximes générales du droit, d'établir des principes féconds ẹ conséquence "). Aussi, lorsque la loi a prononcé, il appartient an pouroir exéculif et à l'autorité administrative, d'assurer l'cxículion des. lois par des prescriptions de détail approprires aux circonstances de temps et de lieux..."

Un autre de nọ maîtres, M. le professeur Berthólenıy qui nous apporte ici le concours de son savoir el de son expérience, se plaignail, il y a vingl ans, que "de plus en plus les lois au lieu l'ètre des énoncés de principe, deviennent des règlements de détails..."

Ces observations générales, vraies de tout temps, se présentent ici avec une force particulière. Ce que vous avez à faire aujourthui, ce n'est pas de préciser une législation antérieure dans un domaine connu ; c'est d'édifier quelque chose de nouveau dans lo domaine législatif. Il faut craindre sur ce terrain nouveau de construire quelque chose de trop rigide dans lequel les faits ne pourront pas tenir. $\Lambda u$ bout d'un certain temps d'épreuve, on s'apercevra qu'il y a des lacunes dans la loi, que télle disposition qui avait paru logique est trop restrictive et difficilement applicable, et, comme c'est la loi qui l'aura créée, il faudra une loi pour l'annuler. Or, l'expérience présente de vingt-trois années, montre qu'il n'est pas toujours facile de faire aboutir une loi.
II s'agit d'ailleurs d'une industric constamment en voie de translormation et de développement : l'industrie électrique; en cẹtte malière, ce qui est vaai aujound'hui ne le sera sans doute plus dans 10 ans, surtout lorsque la loi projelée aura apporlé à cette industrie un instrument de progrès dont clle saura se servir.

Enfin, il y a une raison peut-ètre encore plus forte an poinl de rue pratique pour ne pas faire entrer la loi dans les détails d'application aussi profondément que le tonl les projels prísentés. Un projet comportant bo ou so dispositions, domnera licu in Parlement à des discussions fort longues, suscitera presque inevitablement des anendements. Que lon preme le projel le plus complet, et le mieux étudić de ceux qui sonl présentéc : il ne contieut pas encore loutes les dispositions qu'on aurail pu y mettre. Si on rassemblait toutes les dispositions des différents projels qui nous sont soumis on en trouverait peut-ètre une centaine. Il y a là une source d'anemalentents presque indefinic ; le projet sera renvoyé d’une Chambre à l'allece. Si done, la connmission présente un projel trop complexe, ne risque-l-elle pits d'aller à un échec!

Il me parait indispensable de simplitier les projels de loi en renvoyant à des règlements d'administration publitue toul ae qui peut y être renvoyé.

Je crois assez facile de trouver une base logique de simplilicalion. L'Etal, dans une concession de chule. anra devant lui deux personnes : d'une part, le riverain, proprićlaire de chroits aciluels; d'autre part, le demandeur en concession. A l'ügard du premier, il s'agit de porter alteinte à son droit de propriété ; c'esl un droil nouveau à créer. Le droil actuel du riverain, son droil de propriété va ètre transformé en un troit à indenmité. Cela ne pèul chre fait que par un acte de puissance publique ; l'inlervention du Législateur est indispensable.

Au contrairé, à l'égard du demandeur en concession, la silualion est toute différente. Il viendra dire : soili une chule que je veux aménager. L'Etat répondra : jai le droit de disposer de cette chule, que me donnez-vous en échange !' hous sommes ici dius un domaine purement contractuel. C'est un contrat synallagmalique qui va être passé ; par conséquenl, théoriquement, le législateur pourrail ne pas intervenir dans la détemination des chanses à insérer dans ce contral entre le concessionnaire el l'lital Ilais, si cela est vrai théoriquenont, vous avez fait immédiatement les réserves nécesscitres : il est évident que le Parlernent, qui est le représentajt de la Nation et qui est le dispensaleuir suprème des biens de la Nation, n'admellara pas que le pouvoir exécutif puisse disposer sans aucun contrôle d'une richesse nationale. Il est done toul à fait légilime, je crois, il est tout à fail nécessaire que le Parlement donne des directives au poúvoir exúcutif pour passer ces contrals. Mais, faut-il, pour cela, qu'il entre dans le détail, qu'il prépare presque les contrats lui-mème ? Je ne le pense pas. Il doit se borner à poser des principes el e'est. ensuite aux règlements à préciser les conditions d'applicalion.

Il me semble que, dans cette voic, le Parlemont pent Ironver toutes les garanties désirables que sa volonté sera respectée. D'ail leurs, il s'en faut de peu que le systime que je próconise ne soil in fait accompli. Je demande une foi contenant des principes et renvoyant pour les détails d'application aux règlements d'administration publique. Eh bien, il s'en est fallu de peu que la loi votée successivement par les deux Chambres n'aboutisse : son ćchec a tenu à un amendement, voté au Sénat en deuxième leclure, qui modifiait un point de délail, puis à la fin de la légrislature qui a rendu le projel caduc. Sans quoi nous aurions en vigueur une loi simple qui ne contenait guère que des principes.

D'autre part, il est arrivés qu'so igrit, en igr6 et en igr 7 , le Ministre des Travaux Publics a accordé trois concessions de chutes ; elles ont été réglementées par le Conseil d'Etat dans les décrets de concession, les' cahiers des charges, les règlements d'eau, qui ont précisé tous les détails que nous retrouvons dans les projets soumis à la Commission, le Conseil d'Etat est moralement lié par ces précédents et on pent être certain qu'il insérera des dispositions analogues dans les futurs cahiers des charges. 
Il y a d'ailleurs une manière bien simple d'assurer - si le Parlement le désire - l'application d'un certain nombre de dispositions de détail que le pouvoir exécutif devra respecter, e'est de les préciser dans l'exposé des molifs. Le Conseil d'Etat ne manquera pas de suivre la yoie qui lui sera ainsi tracée el de prendre pour guide la volonté ainsi manifestée par le Gouvernement ot par les Chambres.

Je proposerai donc à la Commission de décider, si elle le juge bon, que ie projel de: loi ne contiendra que des principes : d'abord les principes en vertu desquels seront faites les concessions el qui comporteront moditication, des droits actuels "des liverains, puis un nombre limité de principes directeurs au sujet des contrats à passer entre l'Etal el les concessionnaires.

11 est évident que, dans une maliêre aussi complexe, on a toujours la tentation d'introduire des dispositions nombreuses. te comprends bien la tendance qui a conduit les administrations a proposer des texles très longs; leurs projels sont très étudiés, très intéressants. Mais, si on veul aboutir, il faut se borner, sans quoi, je le répète. je crains un échec. Lá Commission devrait ainsi statuer d'aby rd sur un certain nornbre de dispositions essenlielles qui constitueront la base du projet à établir. J'en indiquicrai quelques-unes. L'une d'olles est la question restée en suspens entre le Sénat et la Chambre.

Quelle est l'autorilé qui concèdera!

Une deuxième question, qui n’avail pas élé envisagée dans les autres projets, mais qui a été examince dans le projel du commerce, est la question des étrangers. Dans quelles conditions pourra-t-on accorder des concessions à des sociétés étrangères oú à des étrangers i) Devra-l-on, 'par exemple, en ce qui concerne les societis, cxiger que les deux liers des administrateurs, seront Frangais. Que dans le Conseil d'administration, la majorité des voix exprincés seront françaises, que le directeur ou le président du Conseil dadministration, l'administrateur-délégué, les commissaires anx comples seront Français el, pour les sociélés en commandite, que le géranl sera Français ?

Troisieme question : quelles seront les charges à imposer aux concessionnaires : Celte question n'a ćlé envisagée que d'une manière sommaire dans le projet de loi volé paŕ les deux Chambres. Taut-il que ces charges suient exciusivement en faveur de l'blat, ou y a-t-il lieu d'en prévoir en faveur des déparlements, des communes, elc... Seront-elles en nature, en argent ? Les redevances seronl-elles fixes ou proportionnelles? Il peut y avoir lieu de prévoir dans certains cas la participation de l'Elat, aux bénéfices. Quand el comment devra s'exercer le contrôle de l'blat, ctc...

Autre difficulte : quel scra le sort des usines existantes? Si l'on décide, par exemple, que dans $7^{5}$ ans elles reviendront à lital, n'y aura-t-il pas la une sorte dexpropriation justifiant l'attribution d'une indemnité

Queslion de l'aide financière de l'Etat : linstallation d'une chule comporte dis grands frais ; d'autre part, elle peut présenter un intérèt public. LEtat interviendra-t-il pécuniairement ? Peuton étudier une organisation du crédit ?

Y aura-t-il lieu d'envisager, comme l'a fail le Ministère de l'Armement, l'application de la loi du 28 mai 19 r5 quj tend à faciliter l'exécution de travanx publics pendant la guerre. Faudrat-il étendre cette application à une période déterminée après la gruerre $3 . . .$.

Il se présente ainsi un cerlain nombre de difficultés, qui devront ètre tranchées avant qu'on ne puisse soumettre un texte à l'examen de la Commission. Ces difficultés tranchées, la procédure la meilleure serait, il me semble, de confier à quelques membres lc soin de rédiger un avant-projet que la Commission examinera.

Reste la question de Poffice ou du Comité dont la création parait s'imposer pour assurer l'unité de vues et d'action que nous désirons obtenir. Vous aurez à vous prononcer sur la création de cet organe, sur sa dénomination : Óffice ou Comité permanent ; sur sa nature (personnalité civile, autonomie, etc...). Pour mon comple, je ne vois pas bien la nécessité de créer un Office autonome avec la personnalité civile. Le principe de la responsabilité ministérielle n'en serait-ils pas atteint? Les attributions de cet organisme à créer palaissent devoir être des attributions consullatives; il donnera des avis soil consultatits quand les ministres le lur demanderont, soil obligatoires quand il sagra de lappr. cation de la loí a voler. Il laudra également determiner la coldposition de cet Olfice ou de ce lomité qui devia comprenure des representants da Parlement, des admiarshations interessées, aes maustriels, des corps savants, el peubetre, des representanis aes interets locaux, par exemple des membres des liliambres ae commerce:

Là encore, d'ailleurs, je crois que la loi doit se borner a poser des règles générales. Cè organisme, évidemment, se' transcomera, s'agrandira al fur el a mesure que se translorineront et s'agraudironl les services de 1 hydraulique. Au fur è à mesure au developpement de lindustrie clectrique, il he taut donc pas, dès à présent, laire de cel organisme à cruer un caure trop rigide qui briderait les progrès ou dèvait éclater.

Messieurs, il me semble quavec la bonme volonté de tous la tiche à accomplir sera relativement lacile; nous devons pouvotr aboutir à bref délai el construire solidement le premier chapith't uu code de Thydraulique. La loi que vous allez préparer aura une indluence considérable sur nos tábrications de guerre et. après la victoire, sur le développement de l'industrie et la próspérité générale du pays.

M. le Présidena: - Voulez-vous me permeltre; Messieurs, d'être l'interprète du Bureau en remerciant lous les orateurs qui nous ont présenté des exposés clairs et lúmineux; $\mathrm{H}$. Petit, les représentants des administratiuns, noon confrère, M. Bougaull et mon ami el collègue, M. Margaine, nous ont fait connaître des vues qui ne sont pas très divergentes.

L'exposé de H. Petit va noús permeltre d'élucider dès aujourd'hui un certain nombre de questions que j'appellerai des ques: lions préjudicielles. Avant d'entrer dans les questions de lond que nous aborderons à la prochaine séance, après avoin mûrement rétléchi, il y a toute une série de questions que nous pouvons útudier tout de suite. Ce sont, à mon avis, les quatre questions suivantes :

$x^{\circ} \mathrm{Y}$ aura-t-il lieu d'élablir une unité de direction 3 On pourra reserver la question de l'attributión à un départemeni ministériel quelconque et la question connexe de savoir si l'organe aura un caractère temporaire ou délinitif, ce qui constitue la deuxième question.

Troisième question, amenée par la seconde : Y a-t-il lieu d'envisager lout de suite un régime définitif ou de faire une légis lation temporaire.

Quatrième question : la loi doit-elle poser seulement des prinvipes ou, au contraire, entrer dans le détaid?

Nous pourrions échanger nos vues sur l'unité dé direction.

M. BúnaRd. - Messieurs, je voudrais poser, d'abond, une question préjudicielle. Je suis très honoré d'appartenir à cette Commission qui comprend des hommes cminents dont noús avons cntendu les exposés lumineux. Mais, je dois vous demander si nous sommos bien d'accord sur ce point, que c'est une Commission consultative, on vue d'un projet gouvernemental, et que les parlementaires qui en font partie ne seront pas liés par lés décisions qui seront prises?

M. Le Président. - C'est l'affaire personnelle de chacun...

M. Bŕrard. - Alors, nous gardons notre liberté individuelle...

M. Le Président - Non, permètlez-moi de vous dire que si nous commençons par réserver notre liberté individuelle, ce n'est pas la peine que nous nous réunissions; que chacun fasse ce qu'il croit devoir faire, mais pour lui-même. Nous n'avons pas i délibérer là-dessus. Que M. Bérand réserve sa liberté... c'est son affaire à lui. Nous sommes jci pour apporter un projet an Gouvernement. Il nous a constitués en arbitres. Par conséquent, nous ne pourrons pas dire : voilà le projét que nous présentons; maik... nou gardons notre liberté.

M. Bérano. - En loute loyaulé, j'estime - et quelques parlementaires pensent peut-ètre comme moi - que, si parfait que soit le projet qui sera élaboré, étant donné les compétences qui sont ici, nous ne pouvons pas, nous Parlementaires, d'avance nous, enchaîner. Voilà ma pensée. 
M. le Président. - C'est l'affaire de chacun.

II. BŕRARd. $-2^{\circ}$ Il est un point qui me semble revenir dans lous les projets, sauf dans celni des Travaux Publics, et aussi dans les conclusions si intéressantes présentées par M. Petit : c'est la création d'un Office national des Forces hydrauliques conslifuant un organe spécial. II me semble que cet organe spécial sill est créé sera une usurpation et sur les droils des ministres el sur ceux du Parlement. Il est incontestable qu'un Office national, irresponsable, quelle que soit la compétence des membres qui en feront partie, aura un rôle, non pas prépondéránt mais ahsolu en matière de forces hydrauliques. Ce será une autorité dressice en face du Parlement.

M. LF PRÉsIDENT. - Vos observations seront faites utilement an moment où nous discuterons la question de l'Office. Nous ne pouvons pas discuter toutes les questions à la fois.

V. Bénum. - Mais, c'est une question qui me paraît prédomi nante. Vous croyez qu'elle ne doit pas venir encore. Je m'incline et je ne la discuterai pas maintenant.

II. Le Président. - Je trouve que la cqueztión de l'office pourra, chre discutée en dernjer lieu parce que tous les principes de législation peuvent être arretés, qu'il y ait un Office ou non. Cette question esl réservée. Je rappelle les questions que je considère comme préjudicielles :

- Utilité de l'unité de direction.

Y a-t-il lieu de réserver l'attribution à un département ministécicl plutôt qu'à un aulre?

- Devons-nous légiféner pour la durée de la guerre, ou pour une période déterminée ou d'une façon définitive.

- La loi doit-elle se contenter de poser des principes, sauf a rewoyer les détails d'application à des règlements d'adminisIration publique.

Toules les autres questions viendront ensuite. Mais celles que Jindique peuvent être tranchées tout de suite el rapidement elles re sont pas gênantes.

M. Bertuéremy. - Dans les questions préjudicielles que vons posez, il y en a une qui coïncide avec celle que soulève M. Bérard Vous demandéz s'il doit y avoir unité de direction. C'est justement en vue d'assurer l'unité de direction que plusieurs projels ont proposé la création d'un office qui aurait la direction de loutes les affaires concernant les forces hydrauliques. C'est une question qu'on pourrait aborder tout de suite.

M. LE Prósidfrt. - L'unité peut être exercée par un ministre, sans qu'il y ait d'office. Le fait que nous posons le principe de l'unité de direction ne crée en rien un préjugé en faveur de l'înstitution d'un Office.

M. Berthelemy. - Parfailement; seulemenl nous abordons fout de suite la question la plus épineuse? S’il ne s'agit que de lire que nous désirons l'unité de direction. nous sommes évideminent unanimes.

M. r.e Président, - II n'est pas mauvais de le proclamer.

M. Berthélemy, - Mais, comment réaliser cetle direction, nous ne sommes pas d'accord.

Il me semble que, dans tout cé qui a été dit, il y a un certain nombre de points qui ont élé unanimement accèptés ou presque Le premier point est celui-ci : il convient d'attribuer à l'autorité le pouvoir de déterminer l'utilisation des forces hydrauliques. Ce n'est pas la peine de le discuter; il est certain que, après 23 ans de discussion. nous sommes tous tombés d'accord sur ce point qu'il convient d'attribuer à l'Etat le ponvoir de déterminer l'utilisation des forces hydrauliques.

Nous sommes tous d'accord aussi sur la nécessité de faire uno distinction ayant pour base, non pas l'ancienne distinction entre cours d'eau navigables ou non navigables, mais la dimension ou l'importance des chutes à exploiter. Quant à la façọn de mettro à la disposition des industriels le droit d'utiliser les chutes, II. Margaine a soulevé une difficulté qui paraît nous faire rétrograder

M. Le Prísinent. - Mais, c'est le fond que vous abordez là. Je vous assure que nos deux conceptions sont conciliables; mais il y an ordre à déterminer. Au lieu de commencer par chercher a nous mettre d'accord sur ces questions de fond, nous pourrions commencer par accepter les règles sur lesquelles nous sommes d'accord.

Mi. Berthélemy: - Alors, prenons les questions préjudicielles. Ellies peuvent être résolues tout de suite.

M. Le Président. - Je me permets de les poser parce qu olles ne peuvent pas engager les travaux ultérieurs de la Commission d'une façon grave.

II. Léon Perrier. - I. le Président, si j'avais eu a ćtablir les questions préjudicielles, $j$ 'en aurai interverti l'ordre.Je crois que la première à poser serait celle de savoir si nous devons faire me législation provisoire oll définitive parce que suivant qu'on y répondra par oui ou par non, nous pourrons avoir une conceplion différente. Nous serions tous prèts, je crois, a lairc beaucoup de concessions s'il s'agit d'une législation provisoire, nécessitée par la Défense nationale, concessions que nous pourrions refuser une législation définitive.

11. re Présinfent. - Très bien.

Examinons font d'abord la tâche qui nous a été assignée. Dans lidée du Gourernement el lans notre propre penséc, notre lache est-elle limitée, provisoire ou, au contritre, devons-nous ádificr un projel de Joi qui surviva à la guerre et permeltra à l'inlustric de se déwlopper.

M. Berthélemy. - Il me semble, d'abord, quil nous faut Homner à l'Administration de l'Armement les satisfactions qu'ollo réclane, Il y aura done toujours une partie exceplionnelle ef lemporaire dans ec que nous ferons. Nais il semble aussi que M. I: Présitent du Conscil nous a demandé aulre chose et nous devoms par conséquent, metlre d'accord ce que nous dommerons à J'Armement avec les principes généraux de la législation définitive.

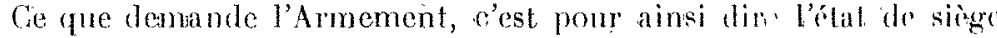
pour les questions de honille blanche. Il faut donner la possibilite l'erproprier, non seulement pour l'btat, mais même pour ceux qui travaillent pour l'Etat. If faut domner cela tout de suite, mais notre rôle ne peul pas se borner là. Nous devons dommer les principes généraux de la loi fulure:

M. LE Présidext. - La proposifion de M. Burlhóleny consiste i nous prononcer en faveur du régime définilif, sous réserve des dispositions qui intéressent, immédiatement la Défense nationate it incorporer soit dans la loi meme, soit dans une loi de finances.

II n'y a pas d'opposition.

M. Léon Perrier. - 11 ne pent y avoir ancune difficulté. Ce qu'il faut à l'Armenent, ec sont des dispositions transitnires of nous, pourrions pent-être, dans lit discussion, commencer par les dispositions transitoires après avoir loutofois élabli les principes sénéraux qui commanderont toute la loj définitive.

M. Berthéceny. - Oui, dans la mesure où elles ne tondicronl pas aux principes généraux.

M. re Présinent: - C'est ủne question, de fond. D'ailleurs, il est possible, pour "armer " l'Armement de mettre ces disposilions transitoires dans un texte spécial a voter-dans la loi de finances.

M. Jéon Pernier. - Etant entendu que ce texle provisoire sora inséré, comme dispositions transitoires dans te texte de loi defnitif. Il faut, par conséquent, parfaitement l'étudier pour qu'il puisse prendre place dans le texle définitif.

M. te Prísident. - C'est entendu.

Voyons le second point. La loi doit-elle poser senlement les principes?

M. Dabat. - Il est certain que nos textes ne posent pas que des principes, mais, lorsque vous arriverez à la rédaction, vous verrez qu'il faudra metlre dans le projet de loi tous les articles que nous y avons mis nous-momes. Thériquement, vol re proposilion est bonne, mais elle n'est pas rólisable.

M. Margaine. - C'est l'évidence même !

M. Brazerx. - To demande à confirmer ro que vient de dire M. Dabat. Fn ce qui nous concerne, nous avons réduit an strict minimum le nombre des articles el je me permettrai de dire it M. Petit que si nous reprenóns les questions qu'il nous a signalées comme étant les plus urgentes, nous arriverons très sensiblement au même nombre d'arideles. Toules ces questions sont prór 
cisément celles que nous avons étudiées et il sera difficile - nous allons néanmoins nous y efforcer -- d'arriver à un texte plus resserré.

M. Bentrélemy. - J'insisterai pourtant pour qu'on adopte la méthode proposée par M. Petil. Copendant, parmi- les dispositons qu'il indique, il cn est qui pourrajent etre insérées dans des règlements d'administration publique, notamment la question des étrangers...

M. re Préstoent. - Non ! Impossible pour tout ce qui touche à la situation des personnes.

M. Mamonne. - Je voubrais demander anx juristes lenr avis sur un point. Je croyais que le Conseil d'Etat ne faisail pas de lois et n'avail pas les pouvoirs d'en faire. Nous ne pouvons done pas lui dire "voilà les principes, vous les methez en loi ". Nous sommes obligés de lui.dire : " vollà ce que devront faire les citoyens, rous lour direz comment ils devront s'y prendre pour le faire ". S'il en est ainsi, je crois que M. Dabal a raison.

II. Benthélem: - Il n'y a pas de donte : le Conscil d'Etat n'a pas le pouvoir de faire des lois.

M. léon Penrur. - le suis partisan d'ume loi extremement precise, tar nous avons pul nous rendre comple bien souvent que les lois faites par le Parlement, sont complèlement déforinés par les règlements d'administration publique on par le Conseil d'Etal. C'est in fail. Combien de fois en effet arons-nous vil l'Administration s'efforcer do modifier la penséc du législateur. Or, dans une matière aussi dúlicate et dilficile, je serais porté à laire préciser dans la loi lous les points qui pourraient donner lien is contestations.

M. Bentréleary. - Oui, mais il ne faut pas que la loj soil Irop louffue, on hien elle perdra lonle espèce de somplesse. D'aillevirs, tout cela est conciliable.

M. PerrT. - M. Margaine me permettra de lui rappeler que son projel de loi n'a que 28 articles.

M. Mangaine. - Et il y en a un qui peut disparaître ; celui yui est relalif à la Commission des forces hydrauliques.

M. Perit. - Nlors, il n'en a même plus que 27 !

M: Manto - M. Je Ministre des Travaux Publics s'était efforcé d'entrer dans la voie indiquée par M. Petil. Nous étions arrivés i ćlablir un projet en dix on douez articles, mais on remettait au Conscil d'Elat la solulion d'une série de questions dont le Parlemont denande de plus en plus à être saisi. Aussi, étant donnée la lendance du Parlement, n'avons-nous pas osé présenter à la Commisșion ce lexte réduit et avons-nous essayć de mettre dans la loi tous les principh's essenticls. Mais je suis tout disporé à me preter a un traval de condensalion si la Commission le désiri.

11. Lonon. Penuer. - C'est une question de rédaction...,

II. he Prósident. - Nous chargerons quclques-mes d'entre nous de rédiger le texle el de lo condenser autant que possible: nous nous in remellrons a leur sagesse et ì leir discernement. If ust "cerlainement désirable d'avoir une loi aussi courte que possible, mais aussi très claire qui ne rénroie an Conseil d'Etat que des queslions de pure application et contienne tout ce qui doit ctre soumis à une décision de la souveraineté nationalc. M. Petif avait eu seulement la erainte que la fortilité du Parlement ne produisit beaucoup d'amendements.

Troisieme question : Unité dé direction. - C'est seulement l'affirmalion d'un désir puisque parsonne n'en conteste l'utilité.

M. Dabst, - Monsieur le Président, je vondrais faire romarquer que ce qui se passe achullement ne soulève aucune difficulté. Tout se fait normalenent aussi bien sur les cours d'eau non navirables que sur les cour's d'eau du domaine public. C'est la nouvelle mélhode proposće qui impose la nécessité d'une unité de direction, mais, dans l'exposé que je-vous ai fait, j'ai insisté sur lis difficultés qu'il y aurait à ce que cetle unité de direction fut assurée par l'Agriculture ou paŕ. les 'Travaux Publics. Je crois dvoir démontré la nécessité de créer un organe indépendant de ses deux Administrations. Ce n'est pas, je tiens à le dire, une, question de boutique, mais c'est la question de ne pas sacrifier les intérêts àgricoles. Pour qu’ils no soient pas sacrifiés, il ne faut pas que ce soit les Ingénieurs des Travaux Publics qui statuent; nous avons éprouvé des difficultés fréquentes à ce sujet, car les Ingénieurs ne voient pas les intérèts agricoles ; mon camarade W. Troté m'a souvent fait cette réflexion, en m'assurant qu'il ne voyait bien les intérêts agricoles que depuis qu'il s'en est occupé... C’est pour cela que j’ai demandé un organisme indépendant des deux administrations.

M: Léon Perrier. - Je me demande s’il n'y a pas dans lit dualilé actuclle une certaine unilć. G'est d'une part, le régime des antorisations données par l'Agriculture ; c'est d'autre part le régime des concessions pratiqués par les Travaux Publics, suivant la nature des cours d'eau. Chacun agit dans son domaine, les inconvénients sont minimes: En lait, ce qui me préoccupe heaucoup plus, r'est la question d'unité de légișlation plulôt que celle de l'atribution des pouvoirs. Ce que beaucoup désirent cest. que les chules soient trailées de la mème façn, quelle que soll ba nature des cours d'eau. Jes Ministères lle l'Agriculture ef des Travaux Publics pourraient parfaitement conserver leurs altributions ; la seule difficulté qui pourrait se présenter, et ce serait rare, serait la nécessité de l'accord à intervenir pour lés chutes qui seraient établies à la fois sur le domaine des Travaux Pubrics et sur celui de l'Agriculture, ce qui peul se produire dans certains cas. Mais, nous aurions un intérêt considérable à ce que chaque ministère travaille sur la partie des eaux dont il a la survelllance; il. $y$ a en effet des Ingénieurs des Travaux Publics qui comprenent mal les intérêts de l'Agriculture; il y a des intérếls de navigation qu'on fera difficilcment défendre par les fónctionnaires de l'Agricullure. Personnellement, j'ai eu l'occasion de voir de près les questions de chules d'eau et j'ai cu souvent, toujours même, à défendre, contre les Ingénieurs, les intérèts agricoles qui sont liés souvent aux grandes chules des Alpes, c'est pourquoit. je verrais avec peine passer les forces hydrauliques de cette région entre les mains des Travaux Publics.

M. te Présment. - Voulez-vous réserver la question d'altibution.

M. Ber'héleny, - Il convient de laisser a aux deux Administrations ce qu'elles fonl, chacune dans' leur sphère. Ce n'est pas sur ce point que nous voulons faire l'unité. Il ne s'agit pas de transporter à I'Agriculture ce que font les Travaux Publics on réciproquemenl. Lil division restera nécessaire, mais ce n'est pas. la question. Je demande qu'on maintienne la division entre rivières niavigables et rivières non navigables, mais pas en ce qui concerne la houille blanche. Pour les concessions de chutes d'eau, celte distinction disparaît et comme ces concessions de chules vont pouvoir se faire tantôt sur les rivières navigables, tantôt sur les rivières dépendant des Travaux Publics, il faut absolument que les' doux Administrations puissent collaborer, puissent se présenter leurs observations pour arriver à une solution honorable. Il faut que les chutes soient socialisées comme les chemins de fer. Il faut une organisation méthodique de toules les chutes et c'est le rôle de l'organiṣne, quel qu'il soit : Ministère, Comité, Office, de préparer cette socialisàtion, de préparer une conception d'ensemhle comme on prépare des plans d'aménagement de villes. Quel est l'orane à cróer Je ne le cherche pas. Quelle sera sa composilion ? Nous la déteminerons, mais il faut un organisme. unique, un ministre pourra être chargé de ces questions, mais i) faudra alors que l'autrorisation soit donnée à un ministre déter: miné entouré de conseils. Nous verrons cela plus tard.

M. Dabat. - Getle unité a été réclamée dans tous les projèts présentés.

M. re Présment. - Te voudrais ramener les orateurs à la question envisagée. Pourquoi cette question de l'unité de direction est-elle en discussion pl Parce que l'arrêté du Présiden du Consël dit : "H est créé a la Présidence du Conseil une Commission chargée d'examiner. les textes des avant-projets de loi... établis par les différentes administrations, de les coordonner en vue de la mise au point d'un projet de loi comportant l'unite de dirècion ". Le Gouvernement s'est prononcé et i] nous demande de rédiger un projet dans ce sens. Si, nous avions un avis diver gent, il faudrait le faire connaîre. Ou alors, dişons que nous sommes bien d'accord el nous verrons ensuite les moyens de réalisation. M. Pelit a sagement proposé de róserver la question 
de l'attribution. Nous ne posons que le principe. Nous nous bornons donc aujourd'hui à établir notre accord unamime sur la nécessité de l'unité de direction. (Assentiment).

La Commission décide qu'elle ne se réunira pas le samedi 2 juin, mais que, par contre, elle siègera le mardi, le mercredi af le jeudi de la semaine suivante.

La séance est leyée à r I h. 50 .

\section{SÉANCE DU 5 JUIN 1917 (5 Séance)}

présenls : MM. Krotz, président ; Develde, Léon Perrier, Wargatne, Gérard, Lourties, Berthélemy, Béraro, Roussead, Buazex, Charmeil, Mahieu, Salles, Ciahen, Reville, Boughayer, Pinot, Cordirr, Marlio, Bovgault, du longbors, Conte; Dabat, Troté, Trsserand, Hrinek, Jugy, Duporteil, Périer de Feral, Lavatio, of Forma (remplaçant M. Gallladed), Amrauné, Petit,

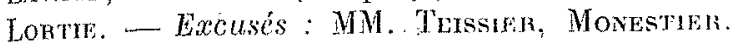

M. le Prúsmenc. - Messieurs, vous ayez sous les yeux le programme de travail que MM. Berthel my et Petil ont bien voulu dresser. Nous allons examiner en premier lieu les principes généralement admis que: je soumeltrai au vole; nous aborderons ensuite les quèstions controversées.

'Tout d'abond, jo lis ee qui se rapporte anx dispositions adoplís. Il a été décidé :

" $7^{\circ}$ Que des dispositions spésiales seraient proposées d'ur"gence, conférant au Ministre de l'Armement les pouvoirs dont "il a besoin.

" $2^{\circ}$ Qu'un lexte définilil devail néanmoins ètre préparé, con* tenant les règles essentielles suivint lesquelles se feronl doré" navanl l'aménagement et l'exploitalion de l'ónergic hyidraulique. " $3^{\circ}$ Que l'unité de direction sux" l'aménagement at l'exploi" taition de l'énergie hydraulique ddevrait être réalisée.

M. Mahmu. - Il me sembule, si mes solvenirs sont exacts, qu'on n'avait pas décidé d'une façon-dófinitive qu'on commencerait par étudier un projet de loi spécial pour le Ministère de l'Armement. Je crois qu'il avait été dit qu'on élablirait le projet do loi définitif et qu'au besoin on extrairait de ce projet définitif les dispositions nécessaires immédialement pour l'Armement, de facon à avoir l'unité de législation, mème dans le provisoire. D'un autre côté, je ne crois pas que cette solution soil de nature à gêner l'Armement dans l'exécution de ses programmes.

-M. le Préstdent. - Nous avons décidé " que des dispositions spéciales seraient proposées d'urgence... i), il n'est pas question de les examiner en premier lieu.

M. Mantev. - Si, d'après ce qui est dit à la page 2.

M. ce Próstonent. - Alors, nous pourrons revenir sur ce point (riand nous arriverons à la page 2.

Je crois que nous sommes d'accond sur le paragraphe $\Lambda$ "dispositions adoptées "?

M. Marrev. - Je ferai toutefois une observation en ce qui encerne cette phrase : il est dit all $2^{\circ}$ qu'wn texte définitif devait néanmoins ĉtre préparé. Il faudrait dire "doit être préparé ".

M. Le Présomsr. - Supprimons " néanmoins". Je contime

B. - "Principes généralement admis à soumettre au vote".

" I I Il convient d'attribuer à l'autorité publique le pouvoir ide déterminer les conditions dans lesquelles seront aménagées ef exploitées les forces hydrauliques naturelles. Les permissions ef concessions conférées par l'autorité auront un caractère essentiellement temporaire is.

M. Cormier. - Il semble que le terme d" " essentiellement temponaine " donne aux concessions un caractère de peu de stabibilité. Il vaudrait mieux dire "durée limitée ".

V. Te Próstont. - C'est la même chosè

M. GÉrard. - Il est dangerenx de dire " essentiellement $1 \mathrm{em}$ noraine s; parce que cela implique que la concession peut être relinée d'un moment à l'autre, tandis que udurée limitée ", c'est
différent.

M. le Président - Il n'y a pas d'opnosition ? Accopté:

M. Bertinkemy. Je voudrais appeler lattertion de la fommission sur ce fait que nous n'avons pas fait un texte de loi, nous n'avons qu'émis des principes el il sera lemps de peser chaque mot lors de la rédaction du projet de loi.

M. Mirgane. - Il y a, dans ce ${ }^{\circ}$, deux choses essentiellement distinctes. La première phrase, qui indique la nócessité d’athribuer à l'autorité publique le pouvoir de déterminer les conditions d'aménagement et d'cxploitation des chutes, ne peut pas soulever d'objection. Il est bien óvident que ce role appartient à l'Etat. Mais, dans la seconde phrase "les permissions et concessions conférées... in il y a quelque chose de lires différent. On consacre un vxai principe : celui de la concession. Il y a concession pare que l'Etat a décida qu'il en serail ainsi. Par conséquent, si nous voulons désomais faire la concession pour la nétallurgie, personne n'aura rien à dire : e'est le fait du Prince. Si la Commission admet ce primcipe, je n'ai plus rien à dire... jr ne wois pas d'inconvénient à ce qu'on concède lout en France.

M. re Présmext. - Revenons à la question. Si les usines sont. concédées, sommes-nous d'accord pour le principe de la durée limitée de la concession? Voilà la question. Pour l'instant, nous n'examinons que le texte el on proposail do romplace "essen tiellement temporaire " par "duréc limitón ". C'est lout. (Aloplé).

W. DAвat - Le mot " permissions " ne correspond à rien. 11 faudrait "r autorisations el concessions "), ec soml les termes cmployés dans les projels.

M. Le Présudent - Vlellons " les auturialions of conecssions conférées " en stupprimant " jar l'aulorilé ".

M. Namev. - Nor's, on réserve la question de savoir s'il faul Clendre la domanialité publique à l'ensemble des conrs d'cau?

M. Berthélemy. - - Si vons le désirez, je suis à volre dinposilion pour discuter sur les théorics.

Trois théries sont en présence : domanialité, propriété de l'blat, un police. La vórité n'est ni du còté de la domanialité ni du côté de la propriété de J'blal. Si vous voulez me perneltre de vous lire l'art. 537 du Code civil : "Les partivuliers ont la libre disposilion des biens qui leur appantiennent, sous les modifications élablics par la Joi "n.

Voici un antre article $4.1 \%$ " La proprićlé est le droil de jonir a de disposer de la maniere la phus ahsolue, pourvu qu'on n'en lasse pas un usage prohibe par les lois ou par les règlements".

Enfin, larticle relatil aux mines fait la mime distinction (el rien ne ressemble plus que lo charbon des mines aux chules

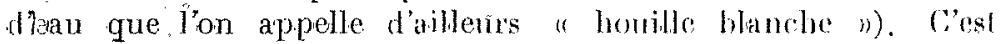
l'art. 552 qui dit :

"La propriété du sol emporte la proprićté du dessus el du dessons.

"Le propriétaire peut fare au-dessus lontes les plantations el constructions qu'il juge à propos, sauf les exceptions établies au litre des Servitudes on Services fonciors.

"Il pent faire au-dessous toutes les constructions el fouilles qu'il jugera à propos, el tirer de ces fouilles lous les produits qu'elles peuvent fournir, sauf les modifications résultant des lois et règlements relatifs aux mines, et des lois ef règloments de police $n$.

Ces trois articles rappellent le droit, de police de l'Etat quand il s'agit de réglementer l'usage de la propriété. Voilà le seul fondement raisonnable de la lógislation que nous voulons préparer.

M. Le Président. - Ce sont des questions que le ródacteur de l'exposé des motifis examinera. On nous a demandé de fajre un texte de loi et non de fonder tout le droit.

M. Manrev. - Mais, ce n'est pas une question de théorie:

M. re Président - Nous devons artêter dos primcipes ef nón pas établir les règles do la propriété sur des bases plus ou moins soljides et controversables.

M. Marred - M. Je Président. c'est une question d'ordre dans le travail... je demandais si on pouvait la discuter.

M. re Présinent. - La Commission appréciera plus tard ce qu'elle doit faire sur ce point. Pronons le paragraphe 2 :

"Le régime applicable aux forces hydrauliques doit varier suivant l'importance do l'énergie aménagée, ef non suivant la qualité juridique du cours d'aù. A tous antres égards, la division 
entre les rivières navigables et celles qui n'ont pas ce caractčre doit être maintenue avec toutes ses conséquences ".

M. Bovcrinyer. - Bien qu'il ne soit pas question de rédaction, il vaudrait mieux employer des termes exacts. "Puissance de l'usine " vaudrait mieux que "énergie aménagée ".

M. Benthélemy. - Je demande la parole, et je sors de mon rôle de professeur de droit. Je n'ai été, en écrivanl ce texte, que le traducteur d'observations qui m'avaient été faites par des ingénieurs. On m'a dit qu'on avail tor't de compter en prenant la force à l'étiage et en prenant le kilowalt comme unité. Je vois que M. Margaine, qui ast ingénieur, n'a pas encouru ce reproche : il n'a pas compté la force à l'éliage, mais la force aménagée. Dé plus, les mêmes ingénjeurs monl décharé qu'il ćlait. d'usage de complar d'après ce qu'on produit, c'est-à-dire en kilowatts, mais que, lorsqu'on mesurait une chule, il valait mieux compter en poncclets, c'est-à-dire d'après ce qui produit.

M. Icón Perrier. - J'appuie tout à fait l'opinion de M. Berthelemy. J'ai participé à nombre de contrats entre des communes de l'ysire et des industriels et, toutes les fois qu'il s'est agi d'une annuité ì verser par l'industriel à la commune, celle annuité a áté calculée daprès la force de la chute afférente aux riverainotés communales ct comptées en poncelels.

M. Perrs. - Je voudrais appuyer ce qu'a dil.M. Benthétemy, Quand on s'exprime en kilowalts, on pense à l'énergie électrique sortant de l'usine. Il vaudrail mieux prendre l'unité de force à l'entrée de l'usine qui n'est pas foncément une usine de production d'énergie électrique, mais qui pent être une papeterie, une scierie.

M. Condrer. - Permettez-moi de dire que poncelet, kilogrammètre, ou autres termes importent peu. C'est toujours la mème chose. La seule différence est que le poncelet vaut too kilogrammòtres, lo cheval $7^{5}$ et le kilowatt environ ro2. La question a été longuement étudiée au Comité d'électricité. J'avais proposé l'adoption du poncelet comme unité de puissance hydraulique : elle a f́té repoussée parce que le poncelet ne représente pas une unité légale. Le kilowatt représenté an contraire une unité déterminée ct s'applique aussí bien à la puissance dectrique qu’à la puissance mécanique ou à la puissance hydraulique, aussi a-t-il ćlé chnisj comme unité devan figurer dans lous les contrats de concession. Mais le kilowatt. le poncelet on le cheval ne mesurent cn ancune façon l'énergie : ils mesurent la puissance. L'énergie se mesure en kilowatts-heiıres et les mots "énergie aménagée " voudraient dire production annuelle. Or, mand on établit une usine. il est impossible de dire cmolle sera sa nroduction annuelle. On pent dire quelle est sa puissance installée sa puissance d'étiage om sa puissance moyenne. Je crois que dans le paragraphe en question, il vaut mieux marler de la puissance de l'usine?

M. ie Présment - Oui. Mettons "puissance de l'usine ") au lien " d'importance de l'énergie aménagée ". (Ascentiment).

" $3^{\circ}$ L'exploitation d'es forces hydrauliques, assimilée à des travaux publics, comportera le droit d'exproprier, Iorsque l'utilité publique aura été déclanée ".

Pas d'opposition ? Adopté !

" $4^{\circ}$ L'exploitation industrielle des forces hydranlicues ne doit être autorisée que sous réserve des intérêts agricoles et de la consorvation des sites pittoresques (Loi du $3 x$ avril rno6) $n$.

M. Manrev. - On ponrrait ajouter " of des intérêts de la navigation $)$.

M. re Présment. - C'est entendu ! nous dirons " des intérêts de. la navigation et de l'agriculture $)$.

" $5^{\circ}$ II y a lien de conserver nour les hesoins éventuels des services publics l'énergic qui peut leur ćfre nécessaire, des mesures étant prises pour qu'en aucun cas les quantifés réservées ne restent inutilisées $"$.

M. Cornrar. - Il me somble que c'est une question de cahier des charges qu'il ne faut pas préciser ici. Je trouve qu'il y a là ruelme chose d'absoh. Ce qu'n doit admettre, c'est me le cahier des charoses détermine une redevance en argent on en nature.

M. re Prósident. - Ciest bien la pensée des rédacteurs. Il est crident cril $v$ a unc mestion de compensation en argent dans To cas où l'ftat renoncerait a bénéfcier de l'énergie.
M. Léon Perrier. - On pourrait dire : " Il y a lieu, s'il est nécessaire, de conserver, etc...

M. BertuÉtsemy. - Messieurs, nous ayons voulu poser uné question et non pas la résoudre. Il nous a semblé que, dans tous lo projets, on se préoccupail de la nécessité que l'Etat peut avoir de réscrver de l'énergic ; qu'adviendrait-il en effet si, toutè l'énergie industrielle étant exploitée, l'Etat n'en avait plus à sa disposition le jour où il voudrait faire de grands travaux? C'est poum cela que nous disons : "én aucun cas, etc..."

Il y a deux questions en présence, deux intérêts à concilier, celui de l'Etat et celui de l'industriel.

M: Léon Perrier. - Deux hypothèses peuvent être envisagées, soit que los besoins des services publics soient immédials, première hypothèse, soit qu'on prévoie, dans le contrat de concession, pour des besoins éventuels, une certaine quantité de force qui sera réservée ot que l'industricl utilisera tant que l'Elat ne la réclamera pas, deuxième hypothèse. C'est bien de la deuxième hypothèse qu'il s'agit?

M. Berthélemy. - Parfaitement.

M. Léon Perrier. - Alors, dans ce cas il n'y a pas lieu de mellre " s'il est nécessaire ". Il est entendu que, dans lous les cas, l'Etat se réservera me pari de force qu'il pourra utiliser quand il le désirera.

M. Pinot. - Je demande quion mettre "réserver ) au lieu de. " conserver". (Accepté).

M. Margarne. - Je voudrais idemander au rédacteur. s'il faudrí inscrire dans la loi l'obligation, pour le cahier des charges, de róserver une fraction d'énergie. Cela peut aller très loin. Si l'industriel est obligé de garder à la disposition des services publics. pour des besoins éventuels, une partie de la force aménagée, les industriels seront dans une incerlitude qui leur rendra la situation impossible. Je comprends qu'on réserve à des hesoins publics une certaine quantité d'énergie à un prix réduit, mais i] faut la fixer et la payer de suite. Si vous imposez à tonites les usines de réserver une partie de force qui peut rester indéfiniment improductive des installations, vous allez faire peser sur celte industrie un poids formidable.

M. Berthébent - Cest ce qui se tronve dans les projets présentés.

M. Condrer, - Je crobs qu'il n'y a aucunie obligation de ce genre dans le projet de M. Margaine. Je n'y suis pas hostile lorsque c'est ulile, mais il me semble que si l'on veut développer l'aménagement des chntes, il faut s'attacher à ne pas alourdir les cabiers des charges de concessions. Il faut que cette clause existe toutes les fois qu'il est nécessaire mais qu'elle soit formulée dans des conditions telles qu'elle n'arrête pas l'industrie. Comme on peut envisager l'aménagement de chutes dans des endroits où il n'y a pas à prévoir de besoins d'énergie pour dés services publics, il y aurait des inconvénients à insérer cette obligation dans la loi. Il ne faut pas oublier que les usines hydrauliques ne se font pas par morceavi ; elles obligent ceux qui les aménagent à engager immédiatement toutes les dépenses dinstallation et à en supporter les charges. Youlons-nous, en dehors de toute nécessité, augmenter ces charges .? Ce serait en même temps augmenter le prix du kilowatt et ciest le contraire de ce que nóns nous proposons, puisque notre but doit être de faire produire beaucoup de kilowatts-heures et de Jes obtenir à bon marché: Il faut donc ócarter ce qui est de nature à augmenter le prix dü kilowatt-heure.

M. Marred - Dans lé projet de loi des Travaux publics on laisse au cahier des charges le soin ide fixer les quantités d'eavet de force à réserver ; c'est d'ailleurs le système qui fonctionne dans les concessions actuelles. I'ajoute qu'elles prévoient toujours pour. les services publics des réductions de tarifs importantes:

M. Jéon Perrier. - Allons-noús mettre cela dáns la Joi ou laisser aux règlements d'administration publique le soin de régler la question?

Il y aurait peut-être lieu, à mon avis de réserver toujours une certaine quantité de force. Nous légiférons; non pas seulement pour le présent, mais encore pour l'avenir. Or, pouvons-nous prévoir les nécéssités futures 9 Si on accorde actuellernent une 
concession sans laire de réserve sur ce point parce quon ne voil pas de besoins présents el que, par la suite, la commune ail pesoin délectricité, dans quelles conditions le concessionnaire st

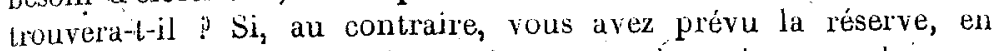
diminuan peut-être l'indemnité due ou la redevance due, en cas d'emploi de cette réserve, vous placerez la collectivilé en meilleurce situalion vis-à-vis du concessionnaire.

M. La Phésident. - Je crois que cesl une question de rédaclion. Voulez-vous me permethe de vous soumellre un texte :

"Des réserves d'Bau ou de lorce pourront ètre introduites dans la cahier des charges pour pourvoir aux besoins éventuels des services publics, des mesures étant prises pour qu'en aucun cas les quantités réservées ne restent inutilisées \#. - (Adopté).

" $6^{\circ}$ Préférence est due aux demandes en concession qui seraient faites par des collectivités administratives pour" le fonclionnement des services publics $)$.

M. Mafulo. - On ne voit pas pourquoi on donne de droit de prélérence aux collectivités adminstratives. Je comprends quion riserve ce droil pour le fonctiomement des services publics, mais jourquoi plútôl quand ces services publics seront gérés par dos colloctivités administratives que s'ils le sonl par dautres : lie qui esi intéressant, c'est que le servicé pubir soit assuré et uon pas qu'il soit assuré par une collectivité administrative plutol quaulrement.

H. Bertó́lemy. - Voilà à quelle idée nous avons oru répondre. l'résentement, quand une collectivité administrative a bosoin de faire des travaux, ellle a à sa disposition l'expropriation. Nous allons mettre l'expropriation à la disposition des industriels, mais nous' réservons la possibilité, pour un service public, de passer avant eux.

M. Mardo. - J'entends bien, mais un service public est une chose el sa gestion en est une autre. Je demande qu'on ne réscrve pas uniquement ce droit aux "collectivilés administralives 1.. Pourquoi pas un concessionnaire, par exemple la Compagnie du Midi.

M. Berthéleny. - G'est l'Etat.

1. Nancio. - Non, c'est un concessionnaire de l'Etat.

M. BFithélemy. - Que nous considérons comme l'Etat. Que te soit l'Elat par lui-mème ou par un concessionnaire de travaux publics, c'est la même chose.

M. Marulo. - Alors, il faudrait ajouter "collectivités administratives ou leurs iconcessionnaires ").

M. Berthélemy. - Vous pariez des concessionnaires de l'ancienne" forme, alors votre formule est inutile parce que, présenlemenl; quand nous disons que l'Etat, les départements ou les communes auront un droit de préférence, nous y comprenons los chemins de fer, nous leur transférons le droit d'expropriation.

M. Marcio. - J'aimerais mieux dire " services publics" d'une façon générale.

1. Berthéleny. -- G’est la même idée.

II. Roussnau. - Je désinerais présenter une observation analogue à cello de M. Marlio. Ce qui fait le caractère de l'utilité publique, c'ost la nature du service et non pas la question de sivoir qui gère le service. Peu importe que ce service soit géré par un industriel ou par une colleclivité administrative. D'autre frirl, si nous laissons le terme de "collectivilés administrativos" ", je erains que nous ne retombions dans les erreurs et les confusions qui ont existé pour lés voies ferrées d'intérêt local et que le Parlement a fait disparaître par la loi de igi3. Nous retrouverons les difficultés résultant dé la superposition des concessions Cles rétrocessions et tous les parlementaires, tous les industriel savent à quelles complications de tous genres conduisent les rótrocessions. Le Parlement a supprimé ces difficultés en votant la loi de igr 3 et nous devons nous en inspinér.

M. Benthélemy: - Alors, supprimons les mots " collectivités administralives $)$ et mettons " en vue d'assurer le fonctionnement des services publics in:-- (Accepté).

M. Bérard. - Si, pour la même opération, il y a concurrence entre une collectivité administrative et un particulier, ne faut-il pas donner la préférence à la collectivité administrative ? Ca n'y est pas.
M. le Président. - La colleclivilé administrative ne peul pas assurer autre chose qu'un service public.

M. Bertuélemy. - La collectivilé administralive ne lravaille que pour un service public. Si elle voulait, par exemple, exproprier pour étendre une maison de rapport qu'elle aurail reçuc cn legs, elle n'aurail pas droil de préférence.

H. Le Préshdext. - Parfailement, elle serait sur le même rang qu'un autre demandeur.

M. BÉRAzD. - Majs, pour celle opération là, d'ordre pour ainsi dire privé, je donnerais la prélérence à la collectivité sur te parliculier parce que les intérêts de la collectivité sont plus intéressants que ceux des particuliers.

H. Bovchayer. - Le cas peut se présenter ainsi : la collectivité administrative réclamant la préférence pour établir une usine de fiaible importance alors qu'un industriel désirerait élablir une usine importante. La collectivite aura-l-elle forcément la préférence?

M. BehtuéLemy. - Je suis entièrement d'accord avee M. Bérasd pour demander que la collectivité passe toujours devant l'inlérèt privé.

M. Mameu, - Je crois que, mème en admetlant la formule de M. Bérard, it faudrait ajouter " pour les besoins de la Défense ualionale ou les besoins généraux du pays ».

H. Berthélemy. - Ce soml au premier ched des services publics !

M. Le Prósident. - Oui, mais ce n'est pas ce qu'on entend généralement par là.

M. Dabat. - Les collectivilés administralives, ce sont les départements el les communes?

M. Bentuélevy. - - Et aussi les syndicats de communes, les Cihambres de Commence, elc.

M. DaBat. - Vous pouvez avoir une commune qui l'asse des travaux daus un intérêt privé.

M. Bentréleay. - Alors, elle ne jouit pas de la prélérence. Elle n'en jouit qu'en lanl que service public.

M. BÉrard. - Permetlez-moi une observation. Vous étendez le droit d'exproprialion aux particuliers, 'droit qu' était jusqu'ici réservé aux collectivités administratives. Dans l'extension de co droit d'expropriation, il se présente à l'esprit que la collectivité doit avoir un droit de préfémence parce qu'elle agit dans l'intérêt de tous, même quand il s'agit d'une opération privée.

M. Gérand. - Si nous faisons tant de réserves, nous pendons de vue le grand principe qui est de produire du kilowatt à bon marché.

M. BÉrard. - Je n'oublie pas ce point de vue, mais je trouve que le département ou la commune doivent passer avant l'industriel. Vous savez bien qu'en fait, le conflit auquel fait allusion II. Gérard ne se produil pas.

Pcusieuns mexbres. - Si, très souvent.

M. Bérard. - Alors, c'est úne raison de plus pour mettre la disposition dans la loj.

M. Pinot. - Te crois que toul le monde est d'accond sur l'idée suivante que, lorsque la collectivité a besoin de fonce, il faut lui donner la préférence. Maintenant, je mé permets, c'est une queslion de rédaction, de soumettre à la commission le point suivant : il ne faudrait pas - car tout est possible - qu'après avoir détruit le barreur particulier, nous laissions les industriels en présence d'un nouveau barrour, qui firenait de da nouvells loi un privilege, et qui pourrait s'opposer, sans motifs, à la création d'une chute. Il y a là une précaution à prendre.

M. Bérard. - Ne portez pas de suspicion pareille contre les collectivités administratives. Il peut' y avoir des abus, mais c'est rare. - (Murmures de protestation).

M. Margaine. - Je voudrais appeler l'attention de la Commission sur des organismes qui, aujourd'hui, ne sont pas très dêveloppés mais qui peuvent devenir plus puissants et je demanderais qu'on les favorisât dans le sens qu'indique M. Bérard. Nous avons des Syndicats agricoles qui font certaines opérations - commerciales d'ailleurs. Ces syndicats ne sont pas encore bien connus ; nous avons besoin de les encourager. Il se peut que ces collectivités qui ne sont pas des collectivités administratives, prennen 
un certain développement. Je demande que la loi permît de leur donner la prélérence lorsque, sérieusement, des collectivités de ce grenre - syndicats agricoles ou autres - veulent faire du commence el pas un service public, veulent par exemple instailer une lromagerle. 11 me semble que cotle prélérence doit leur ètre accordée pour encourager leur naissance ; quand elles se présenleront en même temps qu'un industriel quelconque pour avoir une concession, on devra leur donner la prélérence. Il y a une formule à chercher, nos rédacteurs sauroul bien la trouver.

M. Bovgatir. - Il y a quelque chose qui m'effraie un peu en nia qualité de praticien des chutes. Une collectivité qui n'aura besoin que de peu de force pour le lonctionnement d'un serviee public pourra jeter son dévolu sur une chule d'eau qui pourrail donner des résultats beaucoup plus intéressants. Si vous dites que la préférence est toujours donnée à la collectivité, vous arrivez a arrôter l'industrie hydraulique. Il faudrait dire " aura un droit do próférence à conditions égales ».

M. Berturlenvr. - Parfaitement !

M. Dават. - On oublie une chose, c'est qu'un organe sera créé qui statuera sur lonles ces questions.

ML. Bentuétemy. - Nous parlons du principe el il me semble qu'on doit ajouter en effet a à conditions égales ". C'élail bien notre pensée.

M. Léon Perrarr. - Comme le disait mon collègue Vlargaine, il convient de faire un gros effort pour développer les organisattions colleclivos, surtoul dans les régions déshéritées de nos monbagnes et il faut avoir pour cela ane certaine quantité de force. Wais, je ne.serais pas trop intmansigeant sur la question de la préférence a accorder aux communes pour la concession car, lans l'article que nous avons voté précédemment, on a déjà prévu des réserves fle force au profil des communes. Cela me donne en grande partie satisfaction. Le but à alteindre consiste en lail à fournir de l'énergie à bon marché aux commumes des régions monlagneuses, et à donner à ces communes la possibilité d'organiser des services publics à bas prix. Peu importe le moyen.

M. Le Prúsinerr. - Voulez-vous me permeltre d'essayer ide rapprọhor: les différentes opinions exprimées.

Il senible qu'on'se préociupe lout spécialement, en dehors de l'industrie mêne; du fonctionnement des services publics ; cela est bien entendu, n'en parlons plus. On se préoccupe également das bosoins collectif's ou des besoins de la Défense nationale : disons-le : "II en sera de mème lorsque les besoins de la Défense nationale ou les intérêts collectif's de l'industrie ou de l'agricuture l'exigeront ". Il faudra démontrer que ces besoins ou ces intérêts collectifs existent. Quand il s'agira, par exemple, d'une fromagerie à installer, on devra examiner si son installation répond à des besoins collectil's.

M. Léon Permer. - Dans la plupart de ces cas, on pourra faire jouer le paragraphe précédeñt. Je ne crois pas qu'on puisse honner à des colleclivités, si inléressantés soient-elles, un droit absolu de préférence qui pourrail faire obstacle à l'établissement d'une grande shufe. Il faudrail tenir le plus grand compte de l'observation de M. Bougault.

M. Le Présnnat. - Mais, il n'y a pas dans ce cas a conditions ógales ". "A conditions égales ", cela pourrait s'appliquer à la première formule. Nous sommes bien d'accord pour que le fonctionnement d'un scrvice public passe en première ligne.

UN armbix. - Nous espérons que l'Elat aura la sagesse de ne pas gèner l'installation des chutes.

M. Brazerx. - On pourrait idonner satisfaction à tout le monde en disant : "Compte lenu de l'aménagement d'ensemble de la vallée au poini de vuo de l'intérèt général "). Ce qui importe, c'est l'aménagement d'ensemble de la vallẻe et cela donne satisfaction à toutes les communes et ì toules les collectivités qui se trourent dans cette vallée.

M. Léon Penmer. - Il est certain qu’il ne faudrait pas donner la priorité à des intérèts particuliers qui seraient, pour une grande chute, concurrence avo un service public, si respectable soient res intérêts. Mais si vous avez une grande chute et qu'au milieu de cette derniòre une commune demande à établix une chute réduile, ce serail une chose déplorable que de lui donner dans tous les cas la préférence et de lui accorder ce qu'elile idemande... ('est pour cela que je ne roudrais pas que des colleclivités, mème pour des intérèts généraux puissent dire : : Nous prenons tolle traction de chule ei, cetle fraction de chute, on ne peul pas nous la reluser parce que nous avons un droil de priorité supérieur parce que collectil' à l'intérêt de l'aménagement de la chute tolate $)$.

11. Conbur. - Je m'associe aux observations qui viennent. d'èlre présentées, car il asl certain qu'il ne faul pas gêner l'élablissement des grandes chutes.

Én ce qui concerne de aroil de prélérence lui-mème, j'en vois les inconvénients qui onl élé signalés par M. Bougault et je ne rois pas la nécessité d'inscrire ce droil dáns la loi. Nous sommes. tous d'acerd pour dire qu'il convient que les services publics aient une préférence mais, dautre part, te paragraphe precéden qui prévoit des réserves en laveur des communes; donne satislaction. Enfin, le Ministrc qui doil, concéder ou autoriser a le droil, lorsqu'il se hrouve en présence ide deux demandes, de choisir... peut-on admetre qu'il donnera la prélérence à l'intérèt privé ?

II convient, à l'heure acluelle, d'aménager de grandes chutes. Eh bien, il ny a que les grands services publics, les chemins de fer, qui puissent etre en mesure d'installer de grandes usines; par conséquent, les services publics aulres que ceux-là devront itre desservis par d'aulnes mesures, ćest-à-dire par des réserves failes sur la production des chutes...

M. Margaine. - $I 1$ est bien entendu que, dans notre projel, nous avions parlé du droil de préférence quand il s'agit de deux demandes pour la méne concession. Sil, y a une demande pour. une chnte de 10.000 kilowatis et une demande pour une chute: très fajble, il est évident que ce droit, ne peut pas jouer.

M. Lóon Perlier. - Il y aurail lieu d'apporter beaucoup de précision dans ce lexte. M. Margaine dit qu'il s'agil d'une demande formulée pour la mème chute. Mais, ume chute, c'est parfois chose difficile à déterminer ; une chute peut être fractionnéc en monceaux el tronçons. Quelle sera la siluation de l'Etat lors(Iu'il se trouvera en présence d'une fraction de cours d'eau et de deux dernandes en concession, l'une portant sur une certaine hauteur de chute, l'autre sur la tolalité de da fraction considérée.

M. Margane. - Il sera complètement libre.

M. Léon Pernier. - C'est ce que nous demandons, mais ne dites pas alors dans un texte de loi quil y aura une priorite nócessaire pour une collectivité parce que cela peul etre interprété comme l'obligalion d'acconder à une collectivité parfois très petite la partie de chute qu'elle demande el qui de ce fait fractionnera la chiute totale en trois troncons.

M. Bertuécemy: - Messieurs, ceux (qui auronl à ródiger le lexté définitif tiendront compte de ce qui a été dit, mais il me semble tque vous êtes beaucoup plus d'accond que vous ne le pensez.

Voici la penséc que nous avons eue en préparant ce texte : Deux personnes se présentent pour avoir l'intégralité d'une chute on la même chute, nous lavorisons la commune plulôt qué l'industricl ; si on ne demande pas la mê:" e chule, la question ne se pose pas.

M. Líon Perrier. - On peut ne demander qu'une partie.

M. Berthélemy. - On domnera la préférence au service public: S'il s'ágit d'une chule à aménagè pour l'éclairage d'une villé, qu'un industriel la demande et la commune aussi, nous donnons la préférence à la commune. M. Bongault a fait cette objection (qu'il ne faul pas que la commune devienne " barreur de chute ),

II. Margaine. La question est de savoir si la commune a demandé la chute ou ne l'a pas demandée. Si elle l'a demandéé, clle a la préférence.

M. Berthésemy. - Tout le monde est d'accord.

M. Iéon Perrier. - En pratique, c'est extrèmement difficilé. Vous verrez que si nous n’aboutissons pas a un textc précis basé sur les faits, nous créerons fatalement les conflits.

M. Connrer. - Je crois que l'inscription de cette clause dans la loi nuira beaucoup à l'aménagement des ohutes. Une sociétét 
qui veut établir une chute fait préalablement loutes les études nệccssaires, engage des dépenses. Si vous insérez une clause telle que, lorsqu'elle aura fini ses travaux, elle puisse se voir opposer uu droil de préférence, vous risquez de la décourager. Il me sonlle que l'article précédent suffisait pour donner satislaction aux services publics. Nous sommes d'accord pour ménager les intéréts des services publics, mais nous demandons qu'on n'inlroduise pas une formule qui constituerait un danger pour l'aménagement rationnel des chutes.

II. LE Présideñy. - On préyoit un principe et non la façon do l'appliquer. Peat-être pourrait-on s'exprimer ainsi en ce qui conterne le principe posé :

"Préférence est due aux demandes en concession qui seraient faites pour la même chute en vue d'assurer le fonctionnment d'un service public. La même préférenoe sera accordée lorsque les hesoins de la Défense nationale ou les intérèts collectifs de findustrie on de l'agriculture l'exigeront ". Par conséquent, l'aulorité fera ce qui convient. - (Très bien).

Xons verrons la rédaction dans le texte définitif.

4. Condikr. - Monsieur le l'résident, en ce qui me concerne jo me diclare d acoond sur toute formule qui mettrait simplement en eviaence la nécessité diassurer des services publics, mais je sus opposé à létablissement d'uñe préférence due, obligatoìte. c est une arme dangereuse à mettre dans une loi. Nous demanuons donc qu'on indique la nécessité d'assurer d'abond les serrices publics, mais qu'on ne metle pas ce mot de "prélérence " qui soulèvera toutes espèces ide controverses. En tout cas, si on uecude de le maintenir, il ne pourra être discuté que sur le lexle ichinitil.

11. Margans. - Je ne puis pas comprendre le point de vue ites industriels pance que je crois qu'en fail, les inconvénients no se produiront pas. Nous chemchons à développer les très puissalles chules d'une part, et aider les collectivités d'autre part. (or, quand il s'agira d'une grande chute, jamais une ville ou un syndical ne la demandera. Ce sera toujours un industriel. S'il 5 agit d'une petite chute, elle n'intéressera que le pelit syndical. Par conséquent, l'industriel ne trouvera jamais devant lui le petil syndicat ef inversement.

M. Le Prúsident. - Je demanderai à M. Condier de faire acte de bonme entente et de vouloir bien réserver son opinion définitive pour le moment où nous discuterons le texte mème du projel de loi ; aujourd'hui, nous dirions ce que je proposais tout it l'heure : "Préférence est due aux demandes en concession qui seraient laites, pour la même chuté en vue d'assurea le fonctionnement d'un service public. La même 'próférence sera accurdée lorsque les besoins de la Défense mationale ou les intérêts collectifs de l'industrie on de l'agriculture l'exigeront $"$.

11. Lćon Perrier. - Je crois que nous somnes d'accord. S'il s'agit de la môme chute, les inconvénients que je signalais ne peuvent pas se produire. D'autre part, le mol "l'exigeront ", paraît indiquer qu'il faudra dans tous les cas prouver l'intérêt colleclif. - (Le paragraphe 6 est adopté à l'unanimité).

M. le Prósident. - Paragraphe 7 : "Des dispositions devront assurer aux seuls Français (sauf exception) le bénéfice de l'exploilation des chutes $n$.

Il est entendu, dans l'esprit des rédacteurs, que des exceptions seront prévues dans le texte, notamment en ce qui concerne la catégorie toute spéciale des sociétés. Il y a des sociétés francaises dans lesquelles il peut $\mathrm{y}$ avoir des capitaux, des administrateurs étrangers. Nous ne prenons pas "étrangers "dans le sens d'ennemis, nous ne faisons pas la législation pour le temps do guerre, mais poúr longtemps, peut-être pour toujours si elle est lien faite: Par conséquent, il faut envisager la question, et prévoir des exceptions - à vous de rechercher lesquelles. Le droit de chacun de nous est réservé.

Jo me permettrai de faire tout de suite une suggestion en ce cui concerne les sociétés. Nous pourrions dire que le Président du conseil d'administration, l'Administrateur délćgué, les commisdil Conseil comptes, les censeurs, et les deux tiers des membres dir Conseil seront Francais. En plus de cela, parce que c'est le
Conctionnement mème du Conseil d'administration qui est intéwesant - il faut que la majorité des membres présents soient rança pour que les délibérations soient valables. On pourrail unvisager, en effet, le cas d'un Conseil comprenant neuf' membres dont six françaiş et trois átrangers. Ce Conseil peut délibérer avec cinq membres présents : il ne faudrait pas que, sur ces rinq nembres, il y eut trois ctrangers et deux Françajs !

Il y a aussi la question de naturalisés. Actuellemenl, ils jouissenl des droits des Franģais, mais une proposition de loi demando de réduire ces droits en parcille matière. C'est un point délicat à examiner, car il pourta y avoir un déhal devant la Chambre sil n'est pas mis au point.

11 est évident que nous voulons des eutreprises françaises ; mais nous ne devons pas empêcher l'apport de capitaux étrangers, qui seront représentés quelquefois par dos administrateurs ćlrangers, en minorité d'ailleurs.

M. B́́кAlid. - Vous admeltez que la Société va fonctionner sous la forme de la loi française. Nous avons beaucoup de sociétés sous la forme anglaise.

11. le Prósmbert. - Jous envisageons la forme française; pas les autres.

I. Rovsseav. - Monsieur le Président, bien que je sois d'une manière générale d'accord avec vous sur les principes que vous venez d'énoncer, je me demande si c'est malière à-disposition à introduire dans un texle de loi spécial. Lin réalité, celte question sonlève la question générale du régime ì adopler en ce qui concerne la participation des étrangers à toules les branches de notre activité industrielle et commerciale. Allons-nous la traiter ici ? N'est-ce pas matière à une loi générale ?

Je trouverais même que c'est plulôt matière à cahier des charges el non pas matière à disposition législative. J'indique tout de suite que le danger d'une disposition légisiative, en uri pareil sujel, c'est d'appeler immédiatıment las représailles. Si nons aw...ors d'une manière étroite en ce qui concerne l'admission des etrangers dans nolrc aclivité industrielle, nous provoquons une législation de mème nature dans les pays élrangers. La Commission me dispensera d'insister sur les dangens qu'il pourrail y avoir. Ceux de nos industriels qui sont allés porter au loin l'aclivilé de la France on le droil que nous ne venions pas entraver leur effort en introduisant des mesures restrictives dans notro législation. Je me demande d'ailleurs si, dès maintenant, le Gouvernement n'est pas suffisanment armé. Nous avons décidé que lo Gouvernement était libre de choisir ses concessionnaires el, par de fail nème qu'i] acconde une concession, il fait un contrat avec une personne. Il est done libre d'introduire dans lo contrab toutes les dispositions qu'i] juge utiles, par exemple toutes celles que vient d'indiquer $M$. le Président el qu'il est dès lors inutile de meltre dans un texte de loi. On a déjà procédé de la sorte en beaucoup de matières, notamment pour des entreprises de chemins de fer d'interèt local ou de tramways. On l'a fait pour la Compagnie du Mótropolitain qui est constituée en grande partic par des capilanx belges. On a pris égaloment des disposilions spéciales pour les Compagnies concessionnaires de terriloires au Congo. Je ne crois done pas qu'il y ait nócessite à légiféner el c'est à mon avis dans les cahiers des charges que doivent ôtro insérices les dispositions de cet ondre.

M. Le Présuextr. - Ce que vous dites est tout à fait juste et intéressant. Néanmoins, j'y ferai uno objection.

Etant donné la susceptibilité de l'opinion publique en pareille matière, il est certain que la question sera soulevée an Parlement si nous ne sommes pas allés au devant. Nous aurons des amendements qui n'auronl peut-être pas été suffisamment réfléchis. Nous devons nous y réfléchir ; en tout cas, il faudra que nous introduisions quelques dispositionis dans la loi, peut-être pas tous les détails que j'ai suggérés - c'est plutôt une réforme générale de la loi sur les sociétés qui semblerait s'imposer - mais il faudra que nous disions quelque chose comme ceci : " les chutes seront réservées aux seuls Franģais. Lorsque les, concessions seront faiter à une société qui ne sera pas composée exclusivement de Français. le décret sera rendu en Conseil des Ministres ", ou bien : "sanl exception prévue par décrel rendu en Conseil dea Ministres " 
Ainsi, ce sera la responsabilité ministérielle toute entière qui sera engagée et pas seulement celle du ministre compétent. Le cahier des charges prendra alons les précautions nécessaires dans l'espril que j'indiquai toul à l'heure. Il y a peul-être là une solution qui pourrail satisfaine M. Rousseau. Ce qu'il laut, en tout cas, cost poser le principe pour que la chambre ne puisse pas nous reprocher a avoir negligé la question. Cela pourrail jeter le discredit sur notre travail.

M. housseal. - te sens toute da force de rotre objection, mais jo me demande si ces considérations ne pourraient pas prendre place dans l'exposé des motifs. Il est en effel indispensable qu'on voie que la Commission n'a pas négligé la question. On pourrait dire que la Commission a pensé que, dess maintenant, le Gouvernement est sulfisamment armé, par le fail mème qu'il passe un contral avec le concessionnaire pour y introduire toutes lés précaulions quil juge uliles.

M: Bénand. - Je crois que nolre Président a mille fois raison. Vous n'évitcrez pas le débal devant la Chambre et il y aura très cerlainemeni, surtoul dans les lemps que nous traversons, beaucoup dé Parlementaires pour délendre les droits de la propriété françaisc contre l'invasion ćliangère. Pour mon compte personnel, je suis dans cel étal d'espril el je pense que beaucoup de mes collèguts lo partagent. Il ne s'agit pas de mettre des réflexions dans un exposé des motil's pour faire une loi. Il ne convient pas non plus de laisser' excilusivemenl ce soin au Gouvernement.' Je crois qu'il est indispensable de meltre cette disposition dans la loi el je crois même qu'il faul lè faire d'une façon extrêmement rigide. Il faut que les concessions ne soienl laites qu'aux Français ; on peul admeltre les capitaux étrangers, mais entre les mains des Français. Vous pouve êlre sûrs que l'unanimité de la Chambre ot du Sénat volaront une proposition dans ce sens. Il convient donc de meltre la question au point, entre nous. Je crois que le uanger de réciprocité n'existe pas parce que les pays étrangers cherchent à avoir nos capitaux. On appelle le Hrançais ailleurs, mais, chez nous, on vient prendre. Permettez-moi d'insister pour que rous ne suppirimiéz pas lá proposition de M. le Président. C'est pure prudence vis--à-vis du Parlement.

M. Révilte' - En lait, vous n'aboutirez jamais, à moins de faire une loi disant que toutes les actions doivent être nominalivos. Jo puis vous citer l'exemple d'une société au capital de 15 millions, dont 100.000 franes seulement sont entre les mains des Français. La plupart des membres du Conseil d'ardministralion sonl des hommes de paille ! Or, si la loi n'a pas d'action sur l'industrie en France, i.] faut faire attention quelle n'ait pas de répercussion fâcheuse sur les intérèts français à l'élranger, en Rusisie notamment.

M. LE'Président. - N'oublions pas que nous établissons seulement le principe et que nous disons : "sauf exceptions ". Alors, nos rédacteurs rédigeront le texte, s'inspireront des intérêts très légitimas qui ont été exposés. Je suggérais un décret rendu en Conseil des Ministres... Imaginons que, pour une chute importante, personno ne demande. la concession ot qu'il se troure des śrangers assez handis pour vouloir aménager celle chute. $\mathrm{Ce}$ scrait le Conseil des Ministres qui donnerait son avis et pas seulement lo Ministre compétent.

M. Tisserand. - Je trouve que les mots "sauf exception" paraissent significr qu'au besoin, on donnera la concession à des étrangers.

M. le Président. - Cela n'est pas le texle. Nous ne posons que lo principe. Nous indiqueron's les exceptions et nous dirons qu'clles ne devront pas dépasser certaines limites. Nous verrons cela sur le texte définitif. Je demande à M. Tisserand comme à M. Réville de vouloir bien réserver leurs observations pour le moment où nous verrons ce texte définitif.

M. Léon Perrien. - Toul de mème, il faul retenir l'olservation de M. Réville. Allons-nous faire une loi opérante on non ? Si elle ne doit pas être opérante, mieux vaut s'abstęnir étant donné les répercussions que cela peut avoir dans les législations étrangères.
M. Le Présibent - M. Réville me permeltra de lui dire que son opinion me paraît excessive. Il ne faut pas trancher la question en général en s'appuyant sur le ćas monstrueux. Il y a toujours des hommes de paille, mais ce n'est pas le cas de la majorité des Conseils d'administration.

M. Berthélemy. - Je voudrais faire une observation. Je suis convaincu quil faut poser la question des étrangers, d'abord parce qu'elle sera posée au Parlement et, ensuite, à cause de ce fait que nos forces hydrauliques se trouvent surtout sur les fronlières. Il faul quelque chose de spécial pour les forces hyidrauliques et je trouve que vous avez raison de prévoir un texte. Quel. sera-t-il : Laissons celia à la discussion future...

M. Marlo: - Je trouve quil faut faire très attention, dans cette question de ne pas aller trop loin. La loi que vous ferez ave des restrictions pourra ne rien changer à ce qui se passe. Danś bień des sociélés où les ardministrateuns sont Français, ces administraleurs agissent pour le compte d'autres personnes qui ne paraissent pas el l'inconvénient que nous voyons à cette disposilion proposéc; c'est de gêner par des mesures de réiciprocité le dévelogpement d'affaires où nous avons pris une grande place en Rursie, en Italie, elc.

U. LE Président. - Nous melions " sauf exceplions ". On rédigora en essayant de concilier les intérèls français; à l'intérieur do lia France el au dehors.

M. Privot. - Ne pourrait-on pas mettre l'idée de conventions de réciprocilés?

V. LF Prísidext. - Nos rédacteurs pourront essayer de l'introduire.

M. Conder. - Je voudrais poser une question au sujet des délais pour l'instruction des affaires. Ces délais sont prévus dans phisicurs projets $\mathrm{cl}$, en ce qui nous concerne, ils nous paraissent essentiellement utiles, non pas que, pour une seule administration, nois rencontrions des difficultes, mais nous en rencontrons lorsqu'il y a lieu à communications ontre ministères. On pourrait prévoir un $8^{\circ}$ el diru : " Dés délais seront fixés poúr l'instruction des affaires $)$.

M. LE Présment. - Evidemment, ot les rédacteurs auront à rocherdher les sanctions:

M. Mamreu. - Le projet des Trava urx publics prévoit des délais aveg des sanctions.

W. re Président. - On n'aura qu'à s'y reporter.

Nous arrivons aux questions conlroversées qui seront discutées à la prochaine séance.

J'ai une observation à faire. Jai vu dans la "République de l'Tsène " et idans le' "Journal " des articles se rapportant aux travaux de nolro Commission. Il me semble qu'il vaudrait mieus no faire parter de nos travaux que lorsqu'ils seront términés et jo demande à lous de vouloir bien considérer, pour le moment, nos travaux comme confidentiels.

La séance est levée à midi.

\section{BIBLIOGRAPHIE}

Nous informons nos lecteurs que vient de paraître:

Annuaire de la Houille blanche française, publié par M. An guste Pawlowskr (première année rgr7-rgr8). Fol. de $28 \times 22$ cenl., 184 pages. - Recueil de Notices sur toutes les Sociétés s'occupant des industries de la Houille blanche. - Préface do M. DE LA Brosse, Inspecteur général des Ponts-el-Chaussées. Parrs, 9r, rue Emile-Zola ( $\mathrm{XV}^{\mathrm{e}}$ ).

L'Imprimeur-Gérant . P. LEGENDRE.

Imprimerie pau 1. EG ENDLE \& Cle, 14, rue Beilecordière, iyon. 